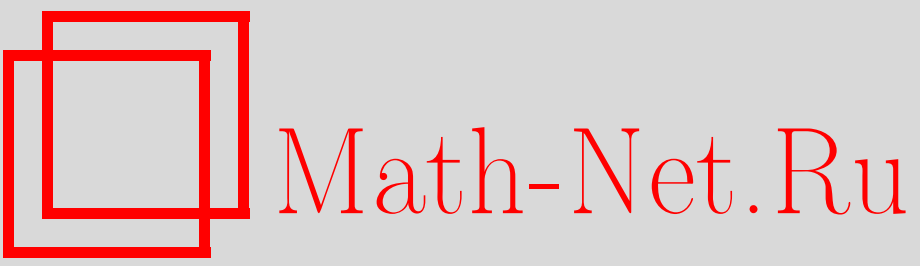

А. Ю. Орлов, Гипергеометрические функции как бесконечносолитонные тауфункции, ТМФ, 2006, том 146, номер 2, 222-250

DOI: https://doi.org/10.4213/tmf2033

Использование Общероссийского математического портала Math-Net.Ru подразумевает, что вы прочитали и согласны с пользовательским соглашением

http://www.mathnet.ru/rus/agreement

Параметры загрузки:

IP: 18.234 .156 .22

26 апреля 2023 г., 15:57:14 
ТЕОРЕТИЧЕСКАЯ

И МАТЕМАТИЧЕСКАЯ

ФИЗИКА

Том 146, № 2

февраль, 2006

(C) 2006 г.

А. Ю. Орлов*

\section{ГИПЕРГЕОМЕТРИЧЕСКИЕ ФУНКЦИИ КАК БЕСКОНЕЧНОСОЛИТОННЫЕ ТАУ-ФУНКЦИИ}

Как известно, резонансные многосолитонные решения зависят от высших времен и набора параметров (интегралов движения). Показано, что солитонные тау-функции уравнений одно- и многокомпонентной цепочек Тода являются тау-функциями дуальной иерархии уравнений, где высшие времена и параметры (интегралы движения) меняются ролями. Многосолитонные решения оказываются рациональными решениями для дуальной иерархии, а бесконечносолитонные тау-функции - тау-функциями гипергеометрического типа дуальной иерархии. Переменные в дуальных иерархиях меняются ролями. Импульсы солитонов связаны с координатами Фробениуса разбиений в разложении рациональных решений по функциям Шура. В качестве примера рассмотрены статсуммы матричных моделей: их ряд теории возмущений, с одной стороны, является гипергеометрической тау-функцией, с другой стороны, может быть интерпретирован как бесконечносолитонное решение.

Ключевые слова: солитоны, рациональные решения, тау-функция, гипергеометрическая функция, дуальность.

\section{1. ВВЕДЕНИЕ}

Гипергеометрическая тау-функция (ГТФ) [1], [2] является обобщением гипергеометрической функции матричного аргумента [3] и определена следующим рядом:

$$
\tau\left(n, \mathbf{t}, T, \mathbf{t}^{*}\right)=c_{n}(T) \sum_{\lambda \in P} \exp \left(\sum_{i=1}^{\infty} T_{n-i}-T_{n+\lambda_{i}-i}\right) s_{\lambda}(\mathbf{t}) s_{\lambda}\left(\mathbf{t}^{*}\right),
$$

где $T=\left\{T_{m}, m=0, \pm 1, \pm 2, \ldots\right\}$ - произвольные параметры, $s_{\lambda}-$ функция Шура, а $P$ - множество всех разбиений. Множитель $c_{n}(T)=\tau(n, \mathbf{0}, T, \mathbf{0})$ в данной работе существенной роли не играет и приведен в приложении А, см. формулы (А.19), (А.20).

Как функция параметров $\mathbf{t}=\left(t_{1}, t_{2}, \ldots\right)$ и $\mathbf{t}^{*}=\left(t_{1}^{*}, t_{2}^{*}, \ldots\right)$ ряд $(1.1)$ является тау-функцией пары иерархий Кадомцева-Петвиашвили (КП), причем $\mathbf{t}$ играет роль

${ }^{*}$ Институт океанологии им. П. П. Ширшова, Москва, Россия. E-mail: orlovs@wave.sio.rssi.ru 
высших времен первой иерархии КП, а t* - времен второй иерархии КП. Как функция параметров $n, \mathbf{t}, \mathbf{t}^{*}$ ряд (1.1) есть тау-функция двумеризованной цепочки Тода (ЦТ). Если переменные $T$ удовлетворяют условиям периодичности $T_{i}=T_{i+N}$, то (1.1) есть тау-функция $N$-периодической ЦТ. Если определенным образом зафиксировать переменные $\mathbf{t}, T$, и $\mathbf{t}^{*}$, можно получить известные гипергеометрические функции многих переменных, введенные в [3]. Также отметим, что ГТФ как формальный ряд совпадает с рядом теории возмущений для некоторых матричных моделей [1], [4]-[6] и используется при построении новых точно решаемых матричных интегралов [7].

Интересно, что для ГТФ можно найти множество приложений. Тау-функции, возникающие в суперсимметричных калибровочных теориях [8], [9], в задачах подсчета чисел Гурвица [10], инвариантов Громова-Виттена на $P^{1}[11]$ и в задачах вычисления индексов пересечения на схемах Гильберта [12], являются примерами ГТФ (см. приложение В к данной работе). Тау-функции, рассмотренные в контексте изучения струн с $c=1$ [13], также принадлежат гипергеометрическому типу.

Данная работа является продолжением и развитием статьи [14] в электронном архиве. Мы изучаем функцию (1.1) как функцию переменных $T$, полагая, что переменные $\mathbf{t}^{*}$ определенным образом зафиксированы (принадлежат к одному из четыpeх семейств, указанных ниже). Показано, что ГТФ (1.1) является многосолитонной тау-функцией некоторой иерархии интегрируемых систем, которую можно назвать дуальной иерархией, и выбор дуальной иерархии определяется выбором $\mathbf{t}^{*}$. Переменные $T$ оказываются высшими временами дуальной иерархии, в роли которой выступает $p$-компонентная двумеризованная ЦТ (более точно, линейными комбинациями этих высших времен, обозначаемых $\tilde{n}^{(m)}, \tilde{\mathbf{t}}^{(m)}, \tilde{\mathbf{t}}^{(m) *}, \quad m=1, \ldots, p$, см. теоремы 1,2$)$.

Для краткости мы будем опускать несущественные аргументы в обозначении ГТФ и писать $\tau\left(\mathbf{t}, T, \mathbf{t}^{*}\right)$ и даже $\tau(\mathbf{t}, T)$ вместо $\tau\left(n, \mathbf{t}, T, \mathbf{t}^{*}\right)$.

Напомним некоторые факты и введем обозначения.

Теория солитонов. Иерархия КП [15], [16] является наиболее популярным примером интегрируемых уравнений. Она состоит из полубесконечного набора нелинейных интегродифференциальных эволюционных уравнений

$$
\partial_{t_{m}} u=K_{m}[u], \quad m=1,2, \ldots,
$$

которые являются коммутирующими потоками: $\left[\partial_{t_{k}}, \partial_{t_{m}}\right] u=0$. Первое нетривиальное уравнение является известным уравнением КП

$$
\partial_{t_{3}} u=\frac{1}{4} \partial_{t_{1}}^{3} u+\frac{3}{4} \partial_{t_{1}}^{-1} \partial_{t_{2}}^{2} u+\frac{3}{4} \partial_{t_{1}} u^{2}
$$

использующимся в физике плазмы и в теории волн на мелкой воде. В настоящее время иерархия КП играет важную роль как в физике, так и в математике. Другим 
важным уравнением является двумеризованная ЦТ

$$
\partial_{t_{1}} \partial_{t_{1}^{*}} \phi_{n}=e^{\phi_{n-1}-\phi_{n}}-e^{\phi_{n}-\phi_{n+1}}
$$

впервые проинтегрированная А. Михайловым [17] и подробно изученная в классической работе [18] в рамках подхода киотской школы [16]. Это уравнение порождает иерархию высших уравнений ЦТ, описывающую потоки по высшим временам, обозначаемым $t_{1}, t_{2}, \ldots$ и $t_{1}^{*}, t_{2}^{*}, \ldots$.

В настоящее время тау-функцию, введенную Сато, следует признать центральным понятием теории солитонов (о тау-функции иерархии КП см. работу [16]). Тау-функция является некоторым универсальным потенциалом, одновременно порождающим иерархии КП и ЦТ. Она зависит от двух полубесконечных наборов времен $t_{1}, t_{2}, \ldots$ и $t_{1}^{*}, t_{2}^{*}, \ldots$ и от дискретной переменной $n: \tau=\tau\left(n, \mathbf{t}, \mathbf{t}^{*}\right)$. Явные формулы таковы [16], [18]:

$$
u=2 \partial_{t_{1}}^{2} \ln \tau\left(n, \mathbf{t}, \mathbf{t}^{*}\right), \quad \phi_{n}\left(\mathbf{t}, \mathbf{t}^{*}\right)=-\ln \frac{\tau\left(n+1, \mathbf{t}, \mathbf{t}^{*}\right)}{\tau\left(n, \mathbf{t}, \mathbf{t}^{*}\right)} .
$$

Рассмотрим три примера тау-функций.

1. Вакуумная тау-функция ЦТ

$$
\tau\left(\mathbf{t}, \mathbf{t}^{*}\right)=\exp \left(\sum_{m=1}^{\infty} m t_{m} t_{m}^{*}\right)
$$

есть простейший пример ГТФ: надо в (1.1) положить $T=0$. Она также является вакуумной тау-функцией иерархии КП, ибо по формуле (1.5) порождает решение $u=0$.

2. $N$-солитонная тау-функция иерархий КП и ЦТ требует задания набора параметров $\left\{p_{i}, q_{i}, a_{i}\right\}, i=1, \ldots, N, p_{i} \neq q_{i}$; каждая пара $\left(p_{i}, q_{i}\right)$ определяет скорость солитона с номером $i$; параметр $a_{i}$ задает начальное положение солитона $i$. Тауфункция $N$-солитонного решения иерархий КП и ЦТ есть

$$
\begin{aligned}
\tau^{\mathrm{sol}}\left(n, \mathbf{t}, \mathbf{t}^{*}\right) & =c\left(\mathbf{t}, \mathbf{t}^{*}\right)\left(\sum_{p=0}^{N} \sum_{1 \leqslant i_{1}<\cdots<i_{p}} \prod_{k<m} \Delta_{i_{k} i_{m}} e^{\eta_{i_{1}}+\cdots+\eta_{i_{p}}}\right)= \\
& =c\left(\mathbf{t}, \mathbf{t}^{*}\right)\left(1+\sum_{i=1}^{N} e^{\eta_{i}}+\sum_{1 \leqslant i<j}^{N} c_{i j} e^{\eta_{i}+\eta_{j}}+\cdots\right),
\end{aligned}
$$

где

$$
\begin{gathered}
\eta_{i}=\xi\left(n, \mathbf{t}, \mathbf{t}^{*}, p_{i}\right)-\xi\left(n, \mathbf{t}, \mathbf{t}^{*}, q_{i}\right)+\ln \frac{a_{i}}{p_{i}-q_{i}}, \\
\xi\left(n, \mathbf{t}, \mathbf{t}^{*}, p\right)=n \ln p+\sum_{m=1}^{\infty} t_{m} p^{m}-\sum_{m=1}^{\infty} t_{m}^{*} p^{-m}
\end{gathered}
$$


а множитель

$$
\Delta_{i j}=\frac{\left(p_{i}-p_{j}\right)\left(q_{i}-q_{j}\right)}{\left(p_{i}-q_{j}\right)\left(q_{i}-p_{j}\right)}
$$

описывает сдвиг фаз взаимодействующих солитонов с номерами $i$ и $j$, равный $\ln \Delta_{i j}$, и где

$$
c\left(\mathbf{t}, \mathbf{t}^{*}\right)=\exp \left(\sum_{m=1}^{\infty} m t_{m} t_{m}^{*}\right)
$$

есть вакуумная тау-функция ЦТ. Для нас представляет интерес бесконечносолитонная тау-функция, отвечающая случаю $N \rightarrow \infty$.

$\mathrm{C}$ точки зрения уравнения КП (1.3) высшие времена $\mathbf{t}^{*}$ есть просто фиксированные параметры, определяющие начальное положение солитона.

ЗАмечАниЕ 1. Отметим, что произвольные дробно-линейные преобразования

$$
p_{i} \rightarrow \frac{a p_{i}+b}{c p_{i}+d}, \quad q_{i} \rightarrow \frac{a q_{i}+b}{c q_{i}+d}, \quad i=1,2, \ldots,
$$

не меняют значения множителей

$$
\Delta_{i j}=\frac{\left(p_{i}-p_{j}\right)\left(q_{i}-q_{j}\right)}{\left(p_{i}-q_{j}\right)\left(q_{i}-p_{j}\right)}, \quad i, j=1,2, \ldots,
$$

входящих в формулу для многосолитонной тау-функции.

В дальнейшем нам будет удобно воспользоваться фермионным представлением для солитонной тау-функции, найденным в работе [16] (обозначения приводятся в приложении А):

$$
\tau^{\mathrm{sol}}\left(n, \mathbf{t}, \mathbf{t}^{*}\right)=\left\langle n\left|e^{H(\mathbf{t})} e^{\sum_{i=1}^{N} a_{i} \psi\left(p_{i}\right) \psi^{*}\left(q_{i}\right)} e^{H^{*}\left(\mathbf{t}^{*}\right)}\right| n\right\rangle .
$$

Наибольшую важность для нас представляет вырожденный случай, описывающий резонансное солитонное решение КП и ЦТ и получающийся из формулы (1.14) при отождествлении некоторых $p_{i}$ (и, аналогично, при отождествлении некоторых $q_{i}$ ), как это будет описано ниже формулой (2.20). При этом величина (1.10) становится равной нулю, т.е. сдвиг фазы солитонов в результате взаимодействия стремится к бесконечности, и это называется резонансным взаимодействием. Оно было впервые обнаружено в системе трех волн [19].

3. Полиномиальная тау-функиия, которая описывает рациональные решения ЦТ, найдена Такасаки. Он предъявил общее решение ЦТ в виде двойного ряда от функций Шура по разбиениям (определения см. ниже) [20]:

$$
\tau\left(n, \mathbf{t}, \mathbf{t}^{*}\right)=\sum_{\lambda, \mu} K_{\lambda \mu}(n) s_{\lambda}(\mathbf{t}) s_{\mu}\left(\mathbf{t}^{*}\right),
$$

где коэффициенты $K_{\lambda \mu}$ являются детерминантами некоторых матриц и решениями специальных билинейных уравнений (соотношений Плюккера) [16]. Ряд (1.15) порождает общие решения иерархий КП и ЦТ [20]; рациональные решения содержатся

2 Теоретическая и математическая физика, т. 146, № 2, 2006 г. 
в (1.15). Например, любая редукция (1.15) к полиному дает некоторое рациональное решение иерархий КП и ЦТ (в этом случае $u$ и $\phi_{n}$ в формулах (1.5) становятся рациональными функциями от $\left.t_{1}\right)$.

Можно показать, что ряд (1.1) также является частным случаем общего решения Такасаки (1.15).

Предположим, что ряд (1.1) обрывается и, следовательно, дает рациональное решение иерархии КП. Покажем, что если временами считать $T_{1}, T_{2}, \ldots$, а не $t_{1}, t_{2}, \ldots$, то ряд (1.1) совпадет с рядом (1.7), в котором количество солитонов $N$ определяется максимальным весом входящих в (1.1) диаграмм Юнга. Этот результат удается получить, если параметры t* в формуле (1.1) зафиксированы так, как будет описано ниже (с помощью одной из формул (2.4)-(2.6) или с помощью одной из формул (3.7)$(3.10))$. Отметим, что эти же специализации времен появляются и в других задачах, связанных с ГТФ, например, в связи с гипергеометрическими функциями матричного аргумента [2] или в задачах о случайных матрицах [7].

Вплоть до настоящего времени нам не удалось распространить результаты данной работы на случай произвольных $\mathbf{t}^{*}$ и на случай общего вида ряда (1.15).

Рассматриваемый круг вопросов может оказаться важным для интерпретации производящей функции инстантонов, найденной в работе [8], что и явилось предпосылкой написания данной работы.

Разбиения. Полиномы многих переменных параметризуются разбиениями. Разбиением называется любая последовательность целых неотрицательных чисел, расположенных в невозрастающем порядке:

$$
\lambda=\left(\lambda_{1}, \lambda_{2}, \ldots, \lambda_{r}, \ldots\right), \quad \lambda_{1} \geqslant \lambda_{2} \geqslant \cdots \geqslant \lambda_{r} \geqslant \cdots \geqslant 0
$$

Числа $\lambda_{i}$ называются частями $\lambda$. Число ненулевых частей называется длиной $\lambda$ и обозначается $\ell(\lambda)$. Сумма частей называется весом $\lambda$ и обозначается $|\lambda|$. Если $n=|\lambda|$, мы говорим, что $\lambda$ есть разбиение $n$. Нулевое разбиение (для которого $\left.\lambda_{1}=0\right)$ обозначается 0 .

Диаграмма разбиения (диаграмма Юнга) может быть определена как набор точек (ячеек) $(i, j) \in \mathbb{Z}^{2}$ таких, что $1 \leqslant j \leqslant \lambda_{i}$. Таким образом, диаграмму Юнга можно считать подмножеством элементов матрицы с $l(\lambda)$ строками и с $\lambda_{1}$ столбцами. Диаграмму разбиения $\lambda$ обозначают тем же символом $\lambda$.

Например,

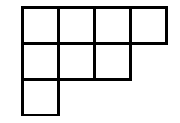

есть диаграмма $(4,3,1)$. Вес этого разбиения равен 8 , его длина равна 3.

Разбиение, диаграмма которого получена транспонированием диаграммы $\lambda$ по отношению к главной диагонали, называется сопряженным разбиением и обозначается $\lambda^{\mathrm{t}}$. 
Есть другое обозначение разбиений, введенное Фробениусом. Предположим, что главная диагональ диаграммы $\lambda$ состоит из $r$ ячеек $(i, i), \quad \alpha_{i}=\lambda_{i}-i$ есть число ячеек в $i$-й строке диаграммы $\lambda$ справа от $(i, i)$ и $\beta_{i}=\lambda_{i}^{\mathrm{t}}-i$ есть число ячеек в $i$-м столбце диаграммы $\lambda$ ниже $(i, i), \quad 1 \leqslant i \leqslant r$. Имеем $\alpha_{1}>\alpha_{2}>\cdots>\alpha_{r} \geqslant 0$ и $\beta_{1}>\beta_{2}>\cdots>\beta_{r} \geqslant 0$. Тогда в обозначениях Фробениуса разбиение $\lambda$ записывается как

$$
\lambda=\left(\alpha_{1}, \ldots, \alpha_{r} \mid \beta_{1}, \ldots, \beta_{r}\right)=(\alpha \mid \beta) .
$$

Можно сказать, что обозначениям Фробениуса соответствует разложение диаграммы $\lambda$ на главные крюки, углы которых находятся на главной диагонали: самый большой крюк - это $\left(\alpha_{1} \mid \beta_{1}\right)$, следующий $\left(\alpha_{2} \mid \beta_{2}\right)$, и так далее до наименьшего крюка $\left(\alpha_{r} \mid \beta_{r}\right)$.

Например, разбиение $(4,3,1)$ состоит из двух крюков $(3,2)$ и $(1,0)$,

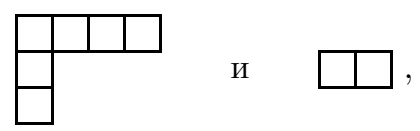

что в обозначениях Фробениуса дает $(3,1 \mid 2,0)$. Если $\lambda=(\alpha \mid \beta)$, то $\lambda^{\mathrm{t}}=(\beta \mid \alpha)$.

ГТФ была введена следующим образом. Рассмотрим функцию $r$, зависящую от $n$, где $n$ - целое число. Для заданного разбиения $\lambda$ положим

$$
r_{\lambda}(x)=\prod_{i, j \in \lambda} r(x+j-i) .
$$

Иными словами, если приписать каждой ячейке $(i, j)$ значение $r(x+j-i)$ и перемножить эти значения по всем ячейкам диаграммы Юнга, то получим $r_{\lambda}(x)$.

Например, если диаграмма разбиения $(4,3,1)$ есть

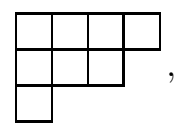

то $r_{\lambda}(x)=r(x+3) r(x+2)(r(x+1))^{2}(r(x))^{2} r(x-1) r(x-2)$. Для нулевого разбиения полагаем $r_{0} \equiv 1$.

В работе [21] было показано, что

$$
\tau_{r}\left(n, \mathbf{t}, \mathbf{t}^{*}\right)=\sum_{\lambda} r_{\lambda}(n) s_{\lambda}(\mathbf{t}) s_{\lambda}\left(\mathbf{t}^{*}\right)
$$

(сумма берется по всем разбиениям, включая нулевое) есть тау-функция уравнений ЦТ и КП. Она была названа ГТФ. К виду (1.1) она приводится заменой

$$
r(m)=e^{T_{m-1}-T_{m}},
$$

т.е. в этом случае $\tau_{r}\left(n, \mathbf{t}, \mathbf{t}^{*}\right)=c_{n}(T) \tau\left(n, \mathbf{t}, T, \mathbf{t}^{*}\right)$. 
Функция Шура $s_{\lambda}(\mathbf{t})$ определяется формулами

$$
s_{\lambda}(\mathbf{t})=\operatorname{det} h_{\lambda_{i}-i+j}(\mathbf{t}), \quad 1 \leqslant i, j \leqslant l(\lambda), \quad \sum_{k=0}^{\infty} z^{k} h_{k}(\mathbf{t})=\exp \left(\sum_{m=1}^{\infty} z^{m} t_{m}\right),
$$

где для $k<0$ полагаем $h_{k}=0$. Полиномы $h_{k}(\mathbf{t})$ называются элементарными функциями Шура, или полностью симметрическими функциями [22].

Взяв в качестве $r$ рациональную функцию, положив $\mathbf{t}^{*}=(1,0,0, \ldots)$ и выбрав $t_{m}=\operatorname{tr} X^{m}, m=1,2, \ldots$, где $X-$ нормальная матрица, получим [1], что ряд (1.20) совпадает с известной гипергеометрической функцией матричного аргумента, см. приложение В.

Уравнение

$$
\partial_{t_{1}} \partial_{t_{1}^{*}} \phi_{n}=r(n) e^{\phi_{n-1}-\phi_{n}}-r(n+1) e^{\phi_{n}-\phi_{n+1}},
$$

(почти совпадающее с ЦТ) имеет решение, выраженное через ГТФ (1.19), вида

$$
\phi_{n}\left(\mathbf{t}, \mathbf{t}^{*}\right)=-\ln \frac{\tau_{r}\left(n+1, \mathbf{t}, \mathbf{t}^{*}\right)}{\tau_{r}\left(n, \mathbf{t}, \mathbf{t}^{*}\right)} .
$$

Тау-функции $\tau_{r}\left(n, \mathbf{t}, \mathbf{t}^{*}\right)$ и $\tau\left(n, \mathbf{t}, T, \mathbf{t}^{*}\right)$ являются решениями уравнения (А.24), приведенного в приложении А, и уравнения (1.4), соответственно. Эти тау-функции отличаются на множитель $c_{n}$, который не зависит от времен $\mathbf{t}, \mathbf{t}^{*}$, поэтому нам достаточно рассмотреть одну из них, например, $\tau\left(n, \mathbf{t}, T, \mathbf{t}^{*}\right)$.

\section{2. ГТФ КАК СОЛИТОННАЯ ТАУ-ФУНКЦИЯ ДУАЛЬНОЙ ИЕРАРХИИ}

Перепишем ГТФ (1.1) в обозначениях Фробениуса. Пусть $\lambda=(\alpha \mid \beta)=\left(\alpha_{1}, \ldots\right.$ $\left.\ldots, \alpha_{k} \mid \beta_{1}, \ldots, \beta_{k}\right)$, где $k$ есть число главных крюков диаграммы $\lambda$. Следуя английским традициям [22], будем называть числа $\alpha_{i}$ руками, а числа $\beta_{i}$ - ногами диаграммы. Перепишем (1.1) как сумму по всем возможным рукам и ногам:

$$
\tau\left(n, \mathbf{t}, T, \mathbf{t}^{*}\right)=c_{n}(T)\left(1+\sum_{\substack{k=1 \\ \alpha_{1}>\cdots>\alpha_{k} \geqslant 0 \\ \beta_{1}>\cdots>\beta_{k} \geqslant 0}}^{\infty} e^{\sum_{i=1}^{k}\left(T_{n-\beta_{i}-1}-T_{n+\alpha_{i}}\right)} s_{(\alpha \mid \beta)}(\mathbf{t}) s_{(\alpha \mid \beta)}\left(\mathbf{t}^{*}\right)\right) .
$$

ЗАмЕчАниЕ 2. Важно отметить, что с помощью предельного перехода $e^{-T_{n+j}} \rightarrow 0$ $\left(e^{T_{n-j}} \rightarrow 0\right)$ можно удалить из суммы $(2.1)$ все члены, содержащие диаграммы, в которых присутствует $j$-я рука ( $j$-я нога). Таким образом, всегда можно заменить суммирование по всем рукам и ногам суммированием по любым наперед заданным наборам рук $S_{\alpha} \subseteq \mathbb{Z}_{\geqslant 0}$ и ног $S_{\beta} \subseteq \mathbb{Z}_{\geqslant}$. Это также означает, что можно рассматривать суммы не по всем разбиениям $P$, а по описанному выше множеству разбиений 
(диаграмм), которое мы будем обозначать $S$. Итак,

$$
\begin{aligned}
& \tau\left(n, \mathbf{t}, T, \mathbf{t}^{*}\right)=c_{n}(T) \sum_{\lambda \in S} e^{\sum_{i=1}^{\infty} T_{n-i}-T_{n+\lambda_{i}-i}} s_{\lambda}(\mathbf{t}) s_{\lambda}\left(\mathbf{t}^{*}\right)= \\
& =c_{n}(T)\left(1+\sum_{k=1}^{\infty} \sum_{\substack{\alpha_{1}>\cdots>\alpha_{k} \geqslant 0 \\
\alpha_{i} \in S_{\alpha}}} \sum_{\substack{\beta_{1}>\cdots>\beta_{k} \geqslant 0 \\
\beta_{i} \in S_{\beta}}} e^{\sum_{i=1}^{k}\left(T_{n-\beta_{i}-1}-T_{n+\alpha_{i}}\right)} s_{(\alpha \mid \beta)}(\mathbf{t}) s_{(\alpha \mid \beta)}\left(\mathbf{t}^{*}\right)\right) .
\end{aligned}
$$

В случае $S \neq P$ для простоты мы будем считать, что $n=0$; общий случай рассмотрим в другой статье.

Если $S_{\alpha}$ и $S_{\beta}$ - конечные множества, тау-функция становится полиномиальной,

$$
\tau\left(n, \mathbf{t}, T, \mathbf{t}^{*}\right)=c_{n}(T)\left(1+\sum_{(\alpha \mid \beta) \subseteq\left(\alpha^{\left.\max \mid \beta^{\max }\right)}\right.} e^{\sum_{i=1}^{k}\left(T_{n-\beta_{i}-1}-T_{n+\alpha_{i}}\right)} s_{(\alpha \mid \beta)}(\mathbf{t}) s_{(\alpha \mid \beta)}\left(\mathbf{t}^{*}\right)\right)
$$

где суммирование ведется по разбиениям, в которых руки и ноги принадлежат заданным множествам, содержащимся в максимальном разбиении $\lambda^{\max }=$ $\left(\alpha^{\max } \mid \beta^{\max }\right)=\left(\alpha_{1}^{\max }, \ldots, \alpha_{k_{\max }}^{\max } \mid \beta_{1}^{\max }, \ldots, \beta_{k_{\max }}^{\max }\right)$.

Введем обозначения

$$
\begin{aligned}
& \mathbf{t}_{\infty}=(1,0,0,0, \ldots), \quad \mathbf{t}(a)=\left(\frac{a}{1}, \frac{a}{2}, \frac{a}{3}, \ldots\right), \\
& \mathbf{t}(\infty, q)=\left(t_{1}(\infty, q), t_{2}(\infty, q), \ldots\right), \quad t_{m}(\infty, q)=\frac{1}{m\left(1-q^{m}\right)}, \quad m=1,2, \ldots, \\
& \mathbf{t}(a, q)=\left(t_{1}(a, q), t_{2}(a, q), \ldots\right), \quad t_{m}(a, q)=\frac{1-\left(q^{a}\right)^{m}}{m\left(1-q^{m}\right)}, \quad m=1,2, \ldots
\end{aligned}
$$

Далее будем считать, что набор $\mathbf{t}^{*}$ принимает вид, указанный в одной из формул (2.4)- (2.6), и тем самым фиксирован.

Лемма 1. Пусть $(\alpha \mid \beta)=\left(\alpha_{1}, \ldots, \alpha_{k} \mid \beta_{1}, \ldots, \beta_{k}\right)$ есть некоторое разбиение, записанное в обозначениях Фробениуса. Тогда

$$
\begin{aligned}
s_{(\alpha \mid \beta)}\left(\mathbf{t}_{\infty}\right) & =\frac{\prod_{i<j}^{k}\left(\alpha_{i}-\alpha_{j}\right)\left(\beta_{i}-\beta_{j}\right)}{\prod_{i, j=1}^{k}\left(\alpha_{i}+\beta_{j}+1\right)} \frac{1}{\prod_{i=1}^{k} \alpha_{i} ! \prod_{i=1}^{k} \beta_{i} !}, \\
s_{(\alpha \mid \beta)}(\mathbf{t}(a)) & =\frac{\prod_{i<j}^{k}\left(\alpha_{i}-\alpha_{j}\right)\left(\beta_{i}-\beta_{j}\right)}{\prod_{i, j=1}^{k}\left(\alpha_{i}+\beta_{j}+1\right)} \prod_{i=1}^{k} \frac{(a)_{\alpha_{i}+1}}{\alpha_{i} !} \prod_{i=1}^{k} \frac{(-1)^{\beta_{i}}(-a)_{\beta_{i}}}{\beta_{i} !} \\
s_{(\alpha \mid \beta)}(\mathbf{t}(\infty, q)) & =\frac{\prod_{i<j}^{k}\left(q^{\alpha_{i}+1}-q^{\alpha_{j}+1}\right)\left(q^{-\beta_{j}}-q^{-\beta_{i}}\right)}{\prod_{i, j=1}^{k}\left(q^{-\beta_{i}}-q^{\alpha_{j}+1}\right)} \frac{1}{\prod_{i=1}^{k}(q ; q)_{\alpha_{i}} \prod_{i=1}^{k}(q ; q)_{\beta_{i}}}
\end{aligned}
$$




$$
\begin{gathered}
s_{(\alpha \mid \beta)}(\mathbf{t}(a, q))=\frac{\prod_{i<j}^{k}\left(q^{\alpha_{i}+1}-q^{\alpha_{j}+1}\right)\left(q^{-\beta_{j}}-q^{-\beta_{i}}\right)}{\prod_{i, j=1}^{k}\left(q^{-\beta_{i}}-q^{\alpha_{j}+1}\right)} \prod_{i=1}^{k} \frac{\left(q^{a} ; q\right)_{\alpha_{i}+1}}{(q ; q)_{\alpha_{i}}} \times \\
\times \prod_{i=1}^{k} \frac{(-1)^{\beta_{i}} q^{(a-1) \beta_{i}}\left(q^{1-a} ; q\right)_{\beta_{i}}}{(q ; q)_{\beta_{i}}} .
\end{gathered}
$$

ДокАЗАТЕЛЬство. Формулы (2.7), (2.8) следуют из формул

$$
\begin{aligned}
s_{(\alpha \mid \beta)}(\mathbf{t}) & =\operatorname{det} s_{\left(\alpha_{i} \mid \beta_{j}\right)}(\mathbf{t}), \quad i, j=1, \ldots, k, \\
s_{\left(\alpha_{i} \mid \beta_{j}\right)}\left(\mathbf{t}_{\infty}\right) & =\frac{1}{\left(\alpha_{i}\right) !\left(\beta_{i}\right) !\left(\alpha_{i}+\beta_{j}+1\right)}, \\
(a)_{(\alpha \mid \beta)} & =\frac{s_{(\alpha \mid \beta)}(\mathbf{t}(a))}{s_{(\alpha \mid \beta)}\left(\mathbf{t}_{\infty}\right)}, \\
(a)_{(\alpha \mid \beta)} & =\prod_{i=1}^{k}(-1)^{\beta_{i}}(a)_{\alpha_{i}+1}(1-a)_{\beta_{i}},
\end{aligned}
$$

где

$$
(a)_{k}:=\frac{\Gamma(a+k)}{\Gamma(a)}=a(a+1) \ldots(a+k-1) .
$$

Формулы (2.9), (2.10) основаны на соотношениях

$$
\begin{aligned}
s_{\left(\alpha_{i} \mid \beta_{j}\right)}(\mathbf{t}(\infty, q)) & =\frac{q^{\left(\beta_{i}^{2}+\beta_{i}\right) / 2}}{(q ; q)_{\alpha_{i}}(q ; q)_{\beta_{i}}\left(1-q^{\alpha_{i}+\beta_{j}+1}\right)} \\
\left(q^{a} ; q\right)_{(\alpha \mid \beta)} & =\prod_{(i, j) \in(\alpha \mid \beta)}\left(1-q^{a+j-i}\right)=\frac{s_{(\alpha \mid \beta)}(\mathbf{t}(a, q))}{s_{(\alpha \mid \beta)}(\mathbf{t}(\infty, q))} \\
\left(q^{a} ; q\right)_{(\alpha \mid \beta)} & =\prod_{i=1}^{k}(-1)^{\beta_{i}} q^{a \beta_{i}-\left(\beta_{i}^{2}+\beta_{i}\right) / 2}\left(q^{a} ; q\right)_{\alpha_{i}+1}\left(q^{1-a} ; q\right)_{\beta_{i}},
\end{aligned}
$$

где

$$
\left(q^{a} ; q\right)_{k}:=\left(1-q^{a}\right)\left(1-q^{a+1}\right) \ldots\left(1-q^{a+k-1}\right), \quad\left(q^{a} ; q\right)_{0}:=1
$$

Рассмотрим другой экземпляр иерархии ЦТ с временами $\tilde{n}, \tilde{\mathbf{t}}, \tilde{\mathbf{t}}{ }^{*}$. Рассмотрим вырожденную (резонансную) бесконечносолитонную тау-функцию этой иерархии в форме

$$
\tau^{\mathrm{sol}}\left(\tilde{n}, \tilde{\mathbf{t}}, \tilde{\mathbf{t}}^{*}\right)=\left\langle\tilde{n}\left|e^{H(\tilde{\mathbf{t}})} \exp \left(\sum_{i \in S_{\alpha}, j \in S_{\beta}} a_{i j} \psi\left(p_{i}\right) \psi^{*}\left(q_{j}\right)\right) e^{H^{*}\left(\tilde{\mathbf{t}}^{*}\right)}\right| \tilde{n}\right\rangle,
$$

где все числа $\left\{p_{i}, i=1,2, \ldots\right\},\left\{q_{i}, i=1,2, \ldots\right\}$ различны и

$$
\tilde{\mathbf{t}}=\left(\tilde{t}_{1}, \tilde{t}_{2}, \tilde{t}_{3}, \ldots\right), \quad \tilde{\mathbf{t}}^{*}=\left(\tilde{t}_{1}^{*}, \tilde{t}_{2}^{*}, \tilde{t}_{3}^{*}, \ldots\right) \text {. }
$$

Если матрица $a_{i j}$ диагональна, число ненулевых матричных элементов равно $N$, то формула (2.20) сведется к формуле (1.14). И наоборот, формулу (2.20) можно 
получить из формулы (1.14) (при $N \rightarrow \infty$ ), если в последней допустить совпадение некоторых чисел $p_{i}$, и, аналогично, некоторых чисел $q_{j}$. Из-за этого вырождения вместо параметров $a_{i}$ формулы (1.14) появляются матричные элементы $a_{i j}$.

Здесь мы не собираемся анализировать поведение функции (2.20), т.е. описывать многосолитонное решение уравнения КП (1.3). Заметим только, что если число ненулевых матричных элементов $a_{i j}$ конечно, то мы получим решение с конечным числом солитонов.

Разложим формулу (2.20) в ряд Тейлора по матричным элементам $a_{i j}$ и используем формулы (А.13) и (А.14) из приложения А:

$$
\begin{aligned}
\tau^{\mathrm{sol}}\left(\tilde{n}, \tilde{\mathbf{t}}, \tilde{\mathbf{t}}^{*}\right) e^{-\sum_{m=1}^{\infty} m \tilde{t}_{m} \tilde{t}_{m}^{*}}= & \\
=1 & +\sum_{k=1}^{\infty} \sum_{\substack{\alpha_{1}>\cdots>\alpha_{k} \geqslant 0 \\
\beta_{1}>\cdots>\beta_{k} \geqslant 0}}\left(\left.\operatorname{det} a_{\alpha_{i} \beta_{j}}\right|_{i, j=1} ^{k}\right) \times \\
& \times\left\langle 0\left|\prod_{i, j=1}^{k} e^{\xi\left(\tilde{n}, \tilde{\mathbf{t}}, \tilde{\mathbf{t}}^{*}, p_{\alpha_{i}}\right)-\xi\left(\tilde{n}, \tilde{\mathbf{t}}, \tilde{t}^{*}, q_{\beta_{j}}\right)} \psi\left(p_{\alpha_{i}}\right) \psi^{*}\left(q_{\beta_{j}}\right)\right| 0\right\rangle .
\end{aligned}
$$

Наконец, с помощью формулы (А.12) получим, что солитонная тау-функция есть

$$
\begin{aligned}
& \tau^{\mathrm{sol}}\left(\tilde{n}, \tilde{\mathbf{t}}, \tilde{\mathbf{t}}^{*}\right)= \\
& \quad=1+\sum_{k=1}^{\infty} \sum_{\substack{\alpha_{1}>\cdots>\alpha_{k} \geqslant 0 \\
\beta_{1}>\cdots>\beta_{k} \geqslant 0}} e^{\xi\left(\tilde{n}, \tilde{\mathbf{t}}, \tilde{\mathbf{t}}^{*}, p_{\alpha_{i}}\right)-\xi\left(\tilde{n}, \tilde{\mathbf{t}}, \tilde{\mathbf{t}}^{*}, q_{\beta_{j}}\right)} \Delta_{(\alpha \mid \beta)}\left(\left.\operatorname{det} a_{\alpha_{i} \beta_{j}}\right|_{i, j=1} ^{k}\right),
\end{aligned}
$$

где суммирование ведется по всем разбиениям $(\alpha \mid \beta)=\left(\alpha_{1}, \ldots, \alpha_{k} \mid \beta_{1}, \ldots, \beta_{k}\right), \quad k=$ $1,2,3, \ldots$, и функции $\xi\left(\tilde{n}, \tilde{\mathbf{t}}, \tilde{\mathbf{t}}^{*}, z\right)$ определены в $(1.9)$. Множитель $\Delta_{(\alpha \mid \beta)}$ отвечает за взаимодействие солитонов:

$$
\Delta_{(\alpha \mid \beta)}=\frac{\prod_{i<j}^{k}\left(p_{\alpha_{i}}-p_{\alpha_{j}}\right)\left(q_{\beta_{i}}-q_{\beta_{j}}\right)}{\prod_{i, j}^{k}\left(p_{\alpha_{i}}-q_{\beta_{j}}\right)\left(q_{\beta_{i}}-p_{\alpha_{j}}\right)} .
$$

Солитонная тау-функция (2.23) зависит от

(а) всех времен $\tilde{n}, \tilde{\mathbf{t}}, \tilde{\mathbf{t}}^{*}$;

(б) матрицы $a_{i j}$, описывающей начальную конфигурацию (взаимное расположение) солитонов, если число ненулевых матричных элементов конечно, то число солитонов конечно;

(в) набора параметров $p_{i}$ и $q_{i}$, определяющих размеры и импульсы солитонов.

Заметим, что правые части формул леммы 1 имеют вид, схожий с правой частью формулы (2.24).

Проверим предположение о том, что (2.23) можно отождествить с (2.2), если в качестве $\mathbf{t}^{*}$ в $(2.2)$ выбран один из наборов $\mathbf{t}_{\infty}, \mathbf{t}(a), \mathbf{t}(\infty, q)$ или $\mathbf{t}(a, q)$. Для этого мы попытаемся отождествить функцию $\xi$, взятую при специальных значениях своего аргумента с набором величин $\left\{T_{n}, n \in \mathbb{Z}\right\}$. Множитель $\operatorname{det} a_{i j}$, учитывающий 
начальную конфигурацию солитонов, отождествим с функцией Шура $s_{(\alpha \mid \beta)}(\mathbf{t})$. Солитонные импульсы $p_{m}$ и $q_{m}$ соотнесем с координатами Фробениуса $\alpha_{m}$ и $\beta_{m}$. В результате получим теорему, которая отображает любую ГТФ (2.3) в некоторую солитонную тау-функцию дуальной иерархии.

ТЕОрема 1. Пусть $\tau\left(n, \mathbf{t}, T, \mathbf{t}^{*}\right)$ задана формулой $(1.1)$, а $\tau^{\mathrm{sol}}\left(\tilde{n}, \tilde{\mathbf{t}}, \tilde{\mathbf{t}}{ }^{*}\right)$ задана формулой (2.20), причем

$$
a_{i j}=a_{i j}(\mathbf{t})=s_{(i \mid j)}(\mathbf{t})
$$

при данном $n$ и заданных наборах $p_{m}, m \in S_{\alpha}, u q_{m}, m+1 \in S_{\beta}$, переменные $\tilde{n}, \tilde{\mathbf{t}}$, $\tilde{\mathbf{t}}^{*}$ связаны с переменными $T$ с помощъю соотношений

$$
\begin{array}{ll}
-T_{n+m}=\sum_{k=1}^{\infty}\left(p_{m}^{k} \tilde{t}_{k}-p_{m}^{-k} \tilde{t}_{k}^{*}\right)+\tilde{n} \ln p_{m}+C_{m+n}, & m \in S_{\alpha}, \\
-T_{n+m}=\sum_{k=1}^{\infty}\left(q_{m}^{k} \tilde{t}_{k}-q_{m}^{-k} \tilde{t}_{k}^{*}\right)+\tilde{n} \ln q_{m}+C_{m+n}, & m+1 \in S_{\beta}
\end{array}
$$

(в этих формулах величинъ $C_{m+n}$ не зависят от $\tilde{n}, \tilde{\mathbf{t}}, \tilde{\mathbf{t}}^{*}$ ); пусть также

$$
\begin{array}{ll}
c_{n}=e^{T_{n-1}+\cdots+T_{1}+T_{0}}=e^{\sum_{m=1}^{n}\left(\sum_{k=1}^{\infty}\left(z_{-m}^{k} \tilde{t}_{k}-z_{-m}^{-k} \tilde{t}_{k}^{*}\right)+\tilde{n} \ln z_{-m}+C_{n-m}\right)}, & n>0, \\
c_{n}=e^{-T_{n}-\cdots-T_{-2}-T_{-1}}=e^{-\sum_{m=0}^{-1-n}\left(\sum_{k=1}^{\infty}\left(z_{m}^{k} \tilde{t}_{k}-z_{m}^{-k} \tilde{t}_{k}^{*}\right)+\tilde{n} \ln z_{m}+C_{m+n}\right)}, & n<0 ;
\end{array}
$$

$c_{0}=1$. Тогда

(A) если

$$
\begin{aligned}
& p_{m}=\frac{a(m+1)+b}{c(m+1)+d}, \quad m \in S_{\alpha} ; \quad q_{m}=\frac{a m+b}{c m+d}, \quad m+1 \in S_{\beta}, \\
& C_{m+n}=-\ln m !, \quad m \in S_{\alpha} ; \quad C_{m+n}=\ln (-m) !, \quad m+1 \in S_{\beta},
\end{aligned}
$$

mo

$$
\tau^{\mathrm{sol}}\left(\tilde{n}, \tilde{\mathbf{t}}, \tilde{\mathbf{t}}^{*}\right) e^{-\sum_{k=1}^{\infty} k \tilde{t}_{k} \tilde{t}_{k}^{*}} c_{n}=\tau\left(n, \mathbf{t}, T, \mathbf{t}_{\infty}\right)
$$

(Б) если

$$
\begin{gathered}
p_{m}=\frac{a(m+1)+b}{c(m+1)+d}, \quad m \in S_{\alpha} ; \quad q_{m}=\frac{a m+b}{c m+d}, \quad m+1 \in S_{\beta}, \\
C_{m+n}=\ln \frac{(a)_{m+1}}{m !}, \quad m \in S_{\alpha} ; \quad C_{m+n}=-\ln \frac{(-1)^{m}(a)_{-m}}{(-m) !}, \quad m+1 \in S_{\beta},
\end{gathered}
$$

mo

$$
\tau^{\mathrm{sol}}\left(\tilde{n}, \tilde{\mathbf{t}}, \tilde{\mathbf{t}}^{*}\right) e^{-\sum_{k=1}^{\infty} k \tilde{t}_{k} \tilde{t}_{k}^{*}} c_{n}=\tau(n, \mathbf{t}, T, \mathbf{t}(a))
$$


(B) если

$$
\begin{gathered}
p_{m}=\frac{a q^{m+1}+b}{c q^{m+1}+d}, \quad m \in S_{\alpha} ; \quad q_{m}=\frac{a q^{m}+b}{c q^{m}+d}, \quad m+1 \in S_{\beta}, \\
C_{m+n}=-\ln (q ; q)_{m}, \quad m \in S_{\alpha} ; \quad C_{m+n}=\ln (q ; q)_{-m}, \quad m+1 \in S_{\beta} .
\end{gathered}
$$

mo

$$
\tau^{\mathrm{sol}}\left(\tilde{n}, \tilde{\mathbf{t}}, \tilde{\mathbf{t}}^{*}\right) e^{-\sum_{k=1}^{\infty} k \tilde{t}_{k} \tilde{t}_{k}^{*}} c_{n}=\tau(n, \mathbf{t}, T, \mathbf{t}(\infty, q)) ;
$$

(Г) $е с л и$

$$
\begin{aligned}
& p_{m}=\frac{a q^{m+1}+b}{c q^{m+1}+d}, \quad m \in S_{\alpha} ; \quad q_{m}=\frac{a q^{m}+b}{c q^{m}+d}, \quad m+1 \in S_{\beta}, \\
& C_{m+n}=\ln \frac{\left(q^{a} ; q\right)_{m+1}}{(q ; q)_{m}}, \quad m \in S_{\alpha} ; \\
& C_{m+n}=-\ln \frac{(-1)^{m} q^{-(a+1) m}\left(q^{1-a} ; q\right)_{-m}}{(q ; q)_{-m}}, \quad m+1 \in S_{\beta},
\end{aligned}
$$

mo

$$
\tau^{\mathrm{sol}}\left(\tilde{n}, \tilde{\mathbf{t}}, \tilde{\mathbf{t}}^{*}\right) e^{-\sum_{k=1}^{\infty} k \tilde{t}_{k} \tilde{t}_{k}^{*}} c_{n}=\tau(n, \mathbf{t}, T, \mathbf{t}(a, q)) .
$$

В этих соотношениях $a, b, c$ и $d$ - комплексные числа, удовлетворяющие условию $a d-b c \neq 0$.

ЗАмечаниЕ 3 . Высшие времена $n, \mathbf{t}, \mathbf{t}^{*}$ исходной иерархии КП являются интегралами движения для второй иерархии ЦТ. И наоборот, высшие времена $\tilde{n}, \tilde{\mathbf{t}}, \tilde{\mathbf{t}}^{*}$ второй иерархии ЦТ, входящие в солитонное решение (2.22), являются интегралами движения исходной иерархии КП. Поэтому естественно назвать эти иерархии дуальными.

Отметим, что иное соотношение дуальности между рациональными и (нерезонансными) солитонными решениями было найдено в работе [23] в контексте изучения систем Калоджеро и Рудженарса: тау-функция рационального решения КП в форме детерминанта Вильсона в этой работе отождествлялась с солитонной тауфункцией уравнения КдФ. Отметим также работы [24], где для одномерной модели Изинга и матричных интегралов рассматривались бесконечносолитонные решения КП с импульсами, лежащими на решетке. Во всех этих работах солитонные решения не были вырожденными (резонансными). Для нашей задачи вырождение играет определяющую роль, позволяя интерпретировать $\operatorname{det} a_{\alpha_{i} \beta_{j}}$ в $(2.22)$ как функцию Шура $s_{\lambda}(\mathbf{t})$.

\section{3. ГТФ КАК СОЛИТОННАЯ ТАУ-ФУНКЦИЯ МНОГОКОМПОНЕНТНОЙ ЦТ}

Мы отошлем читателя к работе [16] и не будем подробно описывать иерархию интегрируемых многокомпонентных уравнений ЦТ, уже нашедшую многочисленные применения. Иерархию $p$-компонентных уравнений ЦТ будем обозначать $p$-ЦТ. 
Приведем вакуумное среднее, являющееся в соответствии с [16] тау-функцией многокомпонентной ЦТ. По аналогии с однокомпонентным случаем мы интерпретируем эту функцию как тау-функцию, описывающую вырожденное (резонансное) солитонное решение многокомпонентной ЦТ:

$$
\begin{aligned}
\tau^{\mathrm{sol}}\left(\tilde{n}^{(1)}, \ldots, \tilde{n}^{(p)} ; \tilde{\mathbf{t}}^{(1)}, \ldots, \tilde{\mathbf{t}}^{(p)} ; \tilde{\mathbf{t}}^{*(1)}, \ldots, \tilde{\mathbf{t}}^{*(p)}\right)= \\
=\langle\tilde{n}| \exp \left(\sum_{m=1}^{p} \sum_{k=1}^{\infty} H_{k}^{(m)} \tilde{t}_{k}^{(m)}\right) \exp \left(\sum_{i \geqslant 0, j>0} a_{i j}^{m n} \psi^{(m)}\left(z_{i}^{(m)}\right) \psi^{*(n)}\left(z_{-j}^{(n)}\right)\right) \times \\
\quad \times \exp \left(\sum_{m=1}^{p} \sum_{k=1}^{\infty} H_{-k}^{(m)} \tilde{t}^{(m)}\right)|\tilde{n}\rangle .
\end{aligned}
$$

В этом выражении $\left(\tilde{t}_{1}^{(m)}, \tilde{t}_{2}^{(m)}, \ldots\right),\left(\tilde{t}_{-1}^{(m)}, \tilde{t}_{-2}^{(m)}, \ldots\right), m=1, \ldots, p$, являются высшими временами иерархий многокомпонентных уравнений ЦТ, а числа

$$
\tilde{n}=\left(\tilde{n}^{(1)}, \ldots, \tilde{n}^{(p)}\right)
$$

суть дискретные переменные $p$-ЦТ, при этом для простоты мы предполагаем, что $\tilde{n}^{(1)}+\cdots+\tilde{n}^{(p)}=0$. В формуле (3.1) "гамильтонианы"

$$
H_{k}^{(m)}=\sum_{n=-\infty}^{+\infty} \psi_{n}^{(m)} \psi_{n+k}^{*}{ }^{(m)}, \quad k \neq 0,
$$

выражаются через многокомпонентные фермионы

$$
\begin{aligned}
\psi_{n}^{(m)} & :=\psi_{n p+m-1}, & \psi_{n}^{*(m)} & :=\psi_{n p+m-1}^{*}, \\
\psi^{(m)}(z) & :=\sum_{k=-\infty}^{\infty} z^{k} \psi_{k}^{(m)}, & \psi^{*(m)}(z) d z & :=\sum_{k=-\infty}^{\infty} z^{-k-1} \psi_{k}^{*(m)},
\end{aligned}
$$

где $\psi_{n}, \psi_{n}^{*}$ определены в приложении А. Верхний индекс $m, m=1, \ldots, p$, фермиона $\psi_{k}^{(m)}, k \in \mathbb{Z}$, мы будем называть цветом этого фермиона.

Это многосолитонное решение $p$-ЦТ задается

(а) набором всех времен $\tilde{n}^{(m)}, \tilde{\mathbf{t}}^{(m)}, \tilde{\mathbf{t}}^{(m)}, m=1, \ldots, p$;

(б) матрицей $a_{i j}^{m n}(i \geqslant 0, j>0, n, m=1, \ldots, p)$, которая определяет начальную конфигурацию многосолитонного решения;

(в) набором $z_{j, m}, j \in \mathbb{Z}, m=1, \ldots, p$, определяющим размеры и импульсы солитонов и сдвиг фаз солитонов при взаимодействии. 
Оказывается, эта тау-функция также может быть отождествленна с ГТФ (2.1), если зафиксировать в ней времена $\mathbf{t}^{*}$ несколько более сложным способом, чем в формулах (2.4)-(2.6).

Рассмотрим такой анзац для высших времен:

$$
\mathbf{t}^{[p]}=(\underbrace{0, \ldots, 0, t_{1}}_{p}, \underbrace{0, \ldots, 0, t_{2}}_{p}, 0, \ldots), \quad \mathbf{t}^{*[p]}=(\underbrace{0, \ldots, 0, t_{1}^{*}}_{p}, \underbrace{0, \ldots, 0, t_{2}^{*}}_{p}, 0, \ldots) .
$$

Введем обозначения

$$
\begin{aligned}
\mathbf{t}_{\infty}^{[p]} & =(\underbrace{0, \ldots, 0,1}_{p}, 0,0, \ldots), \\
\mathbf{t}^{[p]}(a) & =(\underbrace{0, \ldots, 0, \frac{a}{1}}_{p}, \underbrace{0, \ldots, 0, \frac{a}{2}}_{p}, 0, \ldots), \\
\mathbf{t}^{[p]}(\infty, q) & =(\underbrace{0, \ldots, 0, t_{1}(\infty, q)}_{p}, \underbrace{0, \ldots, 0, t_{2}(\infty, q)}_{p}, 0, \ldots), \\
\mathbf{t}^{[p]}(a, q) & =(\underbrace{0, \ldots, 0, t_{1}(a, q)}_{p}, \underbrace{0, \ldots, 0, t_{2}(a, q)}_{p}, 0, \ldots),
\end{aligned}
$$

где $t_{m}(\infty, q)$ и $t_{m}(a, q)$ такие же, как в $(2.5)$ и $(2.6)$.

Тогда на основании результатов приложения Б можно утверждать, что каждая из тау-функций

$$
\tau_{r}\left(n, \mathbf{t}^{[p]}, \mathbf{t}_{\infty}^{[p]}\right), \quad \tau_{r}\left(n, \mathbf{t}^{[p]}, \mathbf{t}^{[p]}(a)\right), \quad \tau_{r}\left(n, \mathbf{t}^{[p]}, \mathbf{t}^{[p]}(\infty, q)\right), \quad \tau_{r}\left(n, \mathbf{t}^{[p]}, \mathbf{t}^{[p]}(a, q)\right)
$$

есть произведение тау-функций, полученных выше в разделе 2.

Чтобы сравнить тау-функцию (3.1) с тау-функциями (3.11), нам понадобятся соотношения, аналогичные тем, что приведены в лемме 1. Сначала введем обозначения. Ниже для записи разбиений используются обозначения Фробениуса. Некоторая их усложненность связана с тем, что нулевое разбиение не имеет записи в обозначениях Фробениуса.

Рассмотрим набор $q$ разбиений, где $0 \leqslant q \leqslant p$, каждое из которых помечено цветом $m_{a}, a=1, \ldots, q$, причем все цвета различны и $1 \leqslant m_{1}<m_{2}<\cdots<m_{q} \leqslant p$. Отметим, что если $q=p$, то $m_{1}=1, m_{2}=2, \ldots, m_{p}=p$.

По этому набору разбиений

$$
\left(\alpha^{\left(m_{a}\right)} \mid \beta^{\left(m_{a}\right)}\right)=\left(\alpha_{1}^{\left(m_{a}\right)}, \alpha_{2}^{\left(m_{a}\right)}, \ldots, \alpha_{k^{\left(m_{a}\right)}}^{\left(m_{a}\right)} \mid \beta_{1}^{\left(m_{a}\right)}, \beta_{2}^{\left(m_{a}\right)}, \ldots, \beta_{k^{\left(m_{a}\right)}}^{\left(m_{a}\right)}\right), \quad a=1, \ldots, q,
$$

строится составное разбиение

$$
(\alpha \mid \beta)=\left(\alpha_{1}, \ldots, \alpha_{k} \mid \beta_{1}, \ldots, \beta_{k}\right), \quad k^{(1)}+\cdots+k^{(q)}=k,
$$

при помощи следующей процедуры. Рассмотрим набор чисел $\left\{\alpha_{k_{i}}^{\left(m_{a}\right)} p+m_{a}-1 \mid i=\right.$ $\left.1, \ldots, k^{\left(m_{a}\right)}, a=1, \ldots, q\right\}$, расположим их в порядке убывания: $\alpha_{1}>\cdots>\alpha_{k}>0$ 
(по построению чисел $\alpha_{k}$ это строго убывающий порядок). Аналогично рассмотрим набор $\left\{\beta_{k_{i}}^{\left(m_{a}\right)} p+m_{a}-p \mid i=1, \ldots, k^{\left(m_{a}\right)}, a=1, \ldots, q\right\}$ чисел, расположенных в порядке убывания: $\beta_{1}>\cdots>\beta_{k}>0$. Это и есть составное разбиение $(\alpha \mid \beta)=$ $\left(\alpha_{1}, \ldots, \alpha_{k} \mid \beta_{1}, \ldots, \beta_{k}\right)$. Можно сказать, что разбиение $(\alpha \mid \beta)$ может быть разложено в разбиения разной окраски из набора цветов $m=1, \ldots, p$. Отметим, что не всякое разбиение может быть разложено таким образом, например, если $p \neq 2$, нельзя разложить разбиение $(\alpha \mid \beta)=(1 \mid 2)$.

Можно доказать следующую лемму (обозначения введены выше).

ЛЕмма 2. Для разбиений $\left(\alpha^{\left(m_{b}\right)} \mid \beta^{\left(m_{b}\right)}\right)=\left(\alpha_{1}^{\left(m_{b}\right)}, \ldots, \alpha_{k^{\left(m_{b}\right)}}^{\left(m_{b}\right)} \mid \beta_{1}^{\left(m_{b}\right)}, \ldots, \beta_{k^{\left(m_{b}\right)}}^{\left(m_{b}\right)}\right)$, где $b=1, \ldots, q$, и составного разбиения $(\alpha \mid \beta)=\left(\alpha_{1}, \ldots, \alpha_{k} \mid \beta_{1}, \ldots, \beta_{k}\right)$

$$
\begin{aligned}
& s_{(\alpha \mid \beta)}\left(\mathbf{t}_{\infty}^{[p]}\right)=(-1)^{\sigma(\alpha, \beta)} \prod_{b=1}^{q} \frac{\prod_{i<j}^{k^{\left(m_{b}\right)}}\left(\alpha_{i}^{\left(m_{b}\right)}-\alpha_{j}^{\left(m_{b}\right)}\right)\left(\beta_{i}^{\left(m_{b}\right)}-\beta_{j}^{\left(m_{b}\right)}\right)}{\prod_{i, j=1}^{k^{\left(m_{b}\right)}}\left(\alpha_{i}^{\left(m_{b}\right)}+\beta_{j}^{\left(m_{b}\right)}+1\right)} \times \\
& \times \frac{1}{\prod_{i=1}^{k^{\left(m_{b}\right)}} \alpha_{i}^{\left(m_{b}\right)} ! \beta_{i}^{\left(m_{b}\right)} !}, \\
& s_{(\alpha \mid \beta)}\left(\mathbf{t}^{[p]}(a)\right)=(-1)^{\sigma(\alpha, \beta)} \prod_{b=1}^{q} \frac{\prod_{i<j}^{k^{\left(m_{b}\right)}}\left(\alpha_{i}^{\left(m_{b}\right)}-\alpha_{j}^{\left(m_{b}\right)}\right)\left(\beta_{i}^{\left(m_{b}\right)}-\beta_{j}^{\left(m_{b}\right)}\right)}{\prod_{i, j=1}^{k^{\left(m_{b}\right)}}\left(\alpha_{i}^{\left(m_{b}\right)}+\beta_{j}^{\left(m_{b}\right)}+1\right)} \times \\
& \times \prod_{i=1}^{k^{\left(m_{b}\right)}} \frac{(a)_{\alpha_{i}^{\left(m_{b}\right)}+1}}{\alpha_{i}^{\left(m_{b}\right)} !} \frac{(-1)^{\beta_{i}^{\left(m_{b}\right)}}(-a)_{\beta_{i}^{\left(m_{b}\right)}}}{\beta_{i}^{\left(m_{b}\right)} !} \\
& s_{(\alpha \mid \beta)}\left(\mathbf{t}^{[p]}(\infty, q)\right)=(-1)^{\sigma(\alpha, \beta)} \prod_{b=1}^{q} \frac{\prod_{i<j}^{k^{\left(m_{b}\right)}}\left(q^{\alpha_{i}^{\left(m_{b}\right)}+1}-q^{\alpha_{j}^{\left(m_{b}\right)}+1}\right)\left(q^{-\beta_{j}^{\left(m_{b}\right)}}-q^{-\beta_{i}^{\left(m_{b}\right)}}\right)}{\prod_{i, j=1}^{k^{\left(m_{b}\right)}}\left(q^{-\beta_{i}^{\left(m_{b}\right)}}-q^{\alpha_{j}^{\left(m_{b}\right)}+1}\right)} \times \\
& \times \frac{1}{\prod_{i=1}^{k^{\left(m_{b}\right)}}(q ; q)_{\alpha_{i}^{\left(m_{b}\right)}}(q ; q)_{\beta_{i}^{\left(m_{b}\right)}}}, \\
& s_{(\alpha \mid \beta)}\left(\mathbf{t}^{[p]}(a, q)\right)=(-1)^{\sigma(\alpha, \beta)} \prod_{b=1}^{q} \frac{\prod_{i<j}^{k^{\left(m_{b}\right)}}\left(q^{\alpha_{i}^{\left(m_{b}\right)}+1}-q^{\alpha_{j}^{\left(m_{b}\right)}+1}\right)\left(q^{-\beta_{j}^{\left(m_{b}\right)}}-q^{-\beta_{i}^{\left(m_{b}\right)}}\right)}{\prod_{i, j=1}^{k^{\left(m_{b}\right)}}\left(q^{-\beta_{i}^{\left(m_{b}\right)}}-q^{\alpha_{j}^{\left(m_{b}\right)}+1}\right)} \times \\
& \times \prod_{i=1}^{k^{\left(m_{b}\right)}} \frac{\left(q^{a} ; q\right)_{\alpha_{i}^{\left(m_{b}\right)}+1}}{(q ; q)_{\alpha_{i}^{\left(m_{b}\right)}}} \frac{(-1)^{\beta_{i}^{\left(m_{b}\right)}} q^{(a-1) \beta_{i}^{\left(m_{b}\right)}}\left(q^{1-a} ; q\right)_{\beta_{i}^{\left(m_{b}\right)}}}{(q ; q)_{\beta_{i}^{\left(m_{b}\right)}}} .
\end{aligned}
$$

Отсюда можно получить обобщение теоремы 1 на случай $p$-ЦТ. Для большей простоты формул положим $n=0$ в ГТФ $\tau\left(n, \mathbf{t}, T, \mathbf{t}^{*}\right)$.

Теорема 2. Пусть тау-функция

$$
\tau^{\mathrm{sol}}\left(\tilde{n}^{(1)}, \ldots, \tilde{n}^{(p)} ; \tilde{\mathbf{t}}^{(1)}, \ldots, \tilde{\mathbf{t}}^{(p)} ; \tilde{\mathbf{t}}^{*(1)}, \ldots, \tilde{\mathbf{t}}^{*(p)}\right)
$$


определена с помощъю формулы (3.1) и параметры выбраны следующим образом:

$$
\begin{gathered}
a_{i j}^{m n}=a_{i j}^{m n}(\mathbf{t})=s_{(i p+m-1 \mid j p+n-1)}(\mathbf{t}), \\
T_{k}^{(m)}=T_{p k+m-1}, \quad m=1, \ldots, p, \\
T_{k}^{(m)}=\sum_{n \neq 0}^{\infty}\left(z_{k, m}\right)^{n} \tilde{t}_{n}^{(m)}-\tilde{n}^{(m)} \ln z_{k, m}-C_{k}^{(m)}, \quad k \in \mathbb{Z},
\end{gathered}
$$

где $C_{k}^{(m)}$ - некоторые константы, которые мы определим ниже.

Тогда

(A)

$$
\begin{aligned}
\tau^{\mathrm{sol}}\left(\tilde{n}^{(1)}, \ldots, \tilde{n}^{(p)} ; \tilde{\mathbf{t}}^{(1)}, \ldots, \tilde{\mathbf{t}}^{(p)} ; \tilde{\mathbf{t}}^{*(1)}, \ldots, \tilde{\mathbf{t}}^{*(p)}\right) \times \\
\\
\times e^{-\sum_{m=1}^{p} \sum_{n=1}^{\infty} n \tilde{t}_{n}^{(m)} \tilde{t}_{n}^{*(m)}}=\tau\left(0, \mathbf{t}, T, \mathbf{t}_{\infty}^{[p]}\right),
\end{aligned}
$$

где левая часть описывает бесконечносолитонное решение с параметрами

$$
\begin{gathered}
z_{k, m}=k+\alpha^{(m)}+1, \quad k \geqslant 0, \quad z_{-k, m}=-k+\alpha^{(m)}, \quad k>0, \quad m=1, \ldots, p, \\
C_{k}^{(m)}=-\ln k !, \quad k \geqslant 0, \quad C_{k}^{(m)}=-\ln (-k) !, \quad k<0
\end{gathered}
$$

(B)

$$
\begin{aligned}
\tau^{\mathrm{sol}}\left(\tilde{n}^{(1)}, \ldots, \tilde{n}^{(p)} ; \tilde{\mathbf{t}}^{(1)}, \ldots, \tilde{\mathbf{t}}^{(p)} ; \tilde{\mathbf{t}}^{*(1)}, \ldots, \tilde{\mathbf{t}}^{*(p)}\right) \times \\
\\
\times e^{-\sum_{m=1}^{p} \sum_{n=1}^{\infty} n \tilde{t}_{n}^{(m)} \tilde{t}_{n}^{*(m)}}=\tau\left(0, \mathbf{t}, T, \mathbf{t}{ }^{[p]}(a)\right)
\end{aligned}
$$

где левая часть описывает бесконечносолитонное решение с параметрами

$$
\begin{aligned}
& z_{k, m}=k+\alpha^{(m)}+1, \quad k \geqslant 0, \quad z_{-k, m}=-k+\alpha^{(m)}, \quad k>0, \\
& C_{k}^{(m)}=\ln \frac{(a)_{k+1}}{k !}, \quad k \geqslant 0, \quad C_{k}^{(m)}=\ln \frac{(-1)^{k}(a)_{-k}}{(-k) !}, \quad k<0 ;
\end{aligned}
$$

(B)

$$
\begin{aligned}
& \tau^{\mathrm{sol}}\left(\tilde{n}^{(1)}, \ldots, \tilde{n}^{(p)} ; \tilde{\mathbf{t}}^{(1)}, \ldots, \tilde{\mathbf{t}}^{(p)} ; \tilde{\mathbf{t}}^{*(1)}, \ldots, \tilde{\mathbf{t}}^{*(p)}\right) \times \\
& \times e^{-\sum_{m=1}^{p} \sum_{n=1}^{\infty} n \tilde{t}_{n}^{(m)} \tilde{t}_{n}^{*(m)}}=\tau\left(0, \mathbf{t}, T, \mathbf{t}^{[p]}(\infty, q)\right),
\end{aligned}
$$

где левая часть описъвает бесконечносолитонное решение с параметрами

$$
\begin{aligned}
& z_{k, m}=q^{k+1}+b^{(m)}, \quad k \geqslant 0, \quad z_{-k, m}=q^{-k}+b^{(m)}, \quad k>0, \\
& C_{k}^{(m)}=-\ln (q ; q)_{k}, \quad k \geqslant 0, \quad C_{k}^{(m)}=-\ln (q ; q)_{-k}, \quad k<0
\end{aligned}
$$

$(\Gamma)$

$$
\begin{aligned}
\tau^{\mathrm{sol}}\left(\tilde{n}^{(1)}, \ldots, \tilde{n}^{(p)} ; \tilde{\mathbf{t}}^{(1)}, \ldots, \tilde{\mathbf{t}}^{(p)} ; \tilde{\mathbf{t}}^{*(1)}, \ldots, \tilde{\mathbf{t}}^{*(p)}\right) \times \\
\\
\times e^{-\sum_{m=1}^{p} \sum_{n=1}^{\infty} n \tilde{t}_{n}^{(m)} \tilde{t}_{n}^{*(m)}}=\tau\left(0, \mathbf{t}, T, \mathbf{t}^{[p]}(a, q)\right),
\end{aligned}
$$


где левая часть описывает бесконечносолитонное решение с параметрами

$$
\begin{gathered}
z_{k, m}=q^{k+1}+b^{(m)}, \quad k \geqslant 0, \quad z_{-k, m}=q^{-k}+b^{(m)}, \quad k>0, \\
T_{k, 0}^{(m)}=\ln \frac{\left(q^{a} ; q\right)_{m+1}}{(q ; q)_{m}}, \quad m \geqslant 0, \quad T_{k, 0}^{(m)}=\ln \frac{(-1)^{k} q^{-(a+1) k}\left(q^{1-a} ; q\right)_{-k}}{(q ; q)_{-k}}, \quad k<0 .
\end{gathered}
$$

В вышеприведенных формулах $\alpha^{(m)}$ и $b^{(m)}$ - произвольные комплексные числа, а обозначения $\mathbf{t}_{\infty}^{[p]}, \mathbf{t}^{[p]}(a), \mathbf{t}^{[p]}(\infty, q)$ и $\mathbf{t}^{[p]}(a, q)$ были введены выше в формулах (3.7)(3.10).

\section{4. МАТРИЧНЫЕ МОДЕЛИ}

\section{КАК БЕСКОНЕЧНОСОЛИТОННЫЕ ТАУ-ФУНКЦИИ}

Как показано в работах [5], [6], ряды теории возмущений для многих матричных моделей описывается тау-функциями гипергеометрического типа. По теоремам 1,2 эти ряды можно интерпретировать как бесконечносолитонную тау-функцию.

4.1. Модель нормальных матриц. Хорошим примером является модель нормальных матриц [25] с аксиально-симметричным взаимодействием. В этом случае согласно [4], [5] ряд теории возмущений является гипергеометрической функцией достаточно общего вида.

Матрица называется нормальной, если она приводится к диагональному виду, или, что то же самое, если она коммутирует со своей эрмитово сопряженной $M^{+}$:

$$
\left[M, M^{+}\right]=0 .
$$

Пусть $M$ диагонализуется унитарной матрицей $U: M=U Z U^{+}, Z=\operatorname{diag}\left(z_{1}, \ldots, z_{n}\right)$, $z_{i}$ - собственные значения $M$. Ясно, что эрмитово сопряженная матрица диагонализуется тем же преобразованием, $M^{+}=U \bar{Z} U^{+}$, причем диагональная матрица $\bar{Z}$ комплексно сопряжена к $Z$.

Статистическая сумма модели нормальных матриц такова:

$$
Z\left(n, \mathbf{t}, \mathbf{t}^{*} ; V\right)=\int d \Omega(M) e^{\operatorname{tr} V_{1}(M)+\operatorname{tr} V_{2}\left(M^{+}\right)+\operatorname{tr} V\left(M, M^{+}\right)},
$$

причем мера интегрирования определена формулой

$$
d \Omega(M)=d_{*} U|\Delta(z)|^{2} \prod_{i=1}^{n} d^{2} z_{i} .
$$

В этом выражении $d_{*} U$ обозначает меру Хаара унитарной группы $U(n)$, а $\Delta(z)-$ определитель Вандермонда,

$$
\Delta(z)=\operatorname{det} z_{i}^{n-k}=\prod_{i<k}^{n}\left(z_{i}-z_{k}\right) .
$$


В случае $n=1$ мы полагаем $\Delta(z)=1$.

Потенциалы $V_{1}(M), V_{2}\left(M^{+}\right)$и $V\left(M, M^{+}\right)$определены с помощью рядов Тейлора:

$$
\begin{aligned}
V_{1}(M) & =\sum_{m=1}^{\infty} t_{m} M^{m}, \quad V_{2}(M)=\sum_{m=1}^{\infty} t_{m}^{*}\left(M^{+}\right)^{m}, \\
V\left(M, M^{+}\right) & =\sum_{k, m=1}^{\infty} v_{k m} M^{k}\left(M^{+}\right)^{m},
\end{aligned}
$$

где $t_{m}, t_{m}^{*}, v_{k m}$ являются комплексными константами связи.

Если перейти от переменных $M$ к переменным $(U, Z)$ и проинтегрировать по $U(n)$, получим

$$
Z\left(n, \mathbf{t}, \mathbf{t}^{*} ; V\right)=C \int_{\mathbb{C}} \ldots \int_{\mathbb{C}}|\Delta(z)|^{2} \prod_{i=1}^{n} e^{V_{1}\left(z_{i}\right)+V_{2}\left(\bar{z}_{i}\right)+V\left(z_{i}, \bar{z}_{i}\right)} d^{2} z_{i},
$$

где интеграл берется по комплексным собственным значениям $z_{i}$, константа $C$ связана с объемом унитарной группы.

Модель нормальных матриц используется при описании капель в квантовом эффекте Холла [26]. В работе [27] было показано, что в частном случае $v_{k m}=\delta_{k, 1} \delta_{m, 1}$ квазиклассический предел $1 / n \rightarrow \infty$ модели нормальных матриц описывает движение границы пятна воды, окруженного пленкой нефти, при наличии источника воды постоянной силы. Эта задача имеет также отношение к некоторым давно поставленным задачам из комплексного анализа [28].

Теперь опишем ряд теории возмущений для статистической суммы (4.6) по константам связи $\mathbf{t}$ и $\mathbf{t}^{*}$. Положим

$$
e^{T_{0}-T_{m}}=A \int_{0}^{+\infty} z^{m} e^{\sum_{k=1}^{\infty} v_{k} z^{k}} d z
$$

Тогда согласно результатам работ [5], [6] имеем следующий асимптотический ряд:

$$
\begin{aligned}
I^{N M}\left(n, \mathbf{t}, \mathbf{t}^{*} ; V\right)= & \tau_{r}\left(n, \mathbf{t}, \mathbf{t}^{*}\right)=I^{N M}(n, 0,0 ; V) \times \\
& \times \sum_{(\alpha \mid \beta)} s_{(\alpha \mid \beta)}(\mathbf{t}) s_{(\alpha \mid \beta)}\left(\mathbf{t}^{*}\right) e^{\sum_{i=1}^{k(\alpha \mid \beta)}\left(T_{n-\beta_{i}-1}-T_{n+\alpha_{i}}\right)} .
\end{aligned}
$$

Если выбрать константы связи $\mathbf{t}^{*}$ в соответствии с одной из формул (3.7)-(3.10), то по теоремам 1, 2 этот ряд теории возмущений является бесконечносолитонной тау-функцией.

В частности, матричный интеграл

$$
I^{N M}\left(n, \mathbf{t}^{(p)}(a), \mathbf{t}^{*(p)}(a) ; V\right)=\int d \Omega(M) e^{\operatorname{tr} U\left(M M^{+}\right)} \operatorname{det}\left(1-M^{p}\right)^{-a} \operatorname{det}\left(1-\left(M^{+}\right)^{p}\right)^{-a},
$$

соответствующий выбору констант связи в виде (3.8), факторизуется в произведение $p$ бесконечносолитонных тау-функций. 
4.2. Двухматричная модель. Рассмотрим двухматричную модель

$$
I^{2 M M}\left(n, \mathbf{t}, \mathbf{t}^{*}\right)=\int e^{\operatorname{tr} V_{1}\left(M_{1}\right)+\operatorname{tr} V_{2}\left(M_{2}\right)} e^{-\operatorname{tr} M_{1} M_{2}} d M_{1} d M_{2},
$$

где $M_{1}$ и $M_{2}$ - эрмитова и антиэрмитова $(n \times n)$-матрицы, соответственно. Мера интегрирования в (4.10) такова:

$$
d M_{1} d M_{2}=\prod_{i=1}^{n} d\left(M_{1}\right)_{i i} d\left(M_{2}\right)_{i i} \prod_{i<j}^{n} d \operatorname{Re}\left(M_{1}\right)_{i j} d \operatorname{Re}\left(M_{2}\right)_{i j} d \operatorname{Im}\left(M_{1}\right)_{i j} d \operatorname{Im}\left(M_{2}\right)_{i j}
$$

Потенциалы определяются константами связи $\mathbf{t}, \mathbf{t}^{*}$ :

$$
V_{1}\left(M_{1}\right)=\sum_{m=1}^{\infty} t_{m} M_{1}^{m}, \quad V_{2}\left(M_{2}\right)=\sum_{m=1}^{\infty} t_{m}^{*} M_{2}^{m}
$$

Как хорошо известно [29], интеграл (4.10) сводится к интегралу по собственным значениям $x_{i}$ и $y_{i}$ матриц $M_{1}$ и $M_{2}$, соответственно.

Имеем

$$
I^{2 M M}\left(n, \mathbf{t}, \mathbf{t}^{*}\right) \frac{(2 \pi)^{n}}{C_{n}}=\sum_{\lambda}(n)_{\lambda} s_{\lambda}(\mathbf{t}) s_{\lambda}\left(\mathbf{t}^{*}\right)
$$

где

$$
(n)_{\lambda}=\prod_{i, j \in \lambda}(n+j-i)=\frac{\Gamma\left(n+1+\lambda_{1}\right) \Gamma\left(n+\lambda_{2}\right) \ldots \Gamma\left(\lambda_{n}\right)}{\Gamma(n+1) \Gamma(n) \ldots \Gamma(1)},
$$

а обьем унитарной группы включен в постоянную $C_{n}[6]$.

Таким образом, если в этом выражении выбрать константы связи в соответствии с одной из формул (3.7)-(3.10), статсумму (4.10) можно интерпретировать как бесконечносолитонное решение из теорем 1,2 .

Отметим, что на уровне ряда теории возмущений матричный интеграл

$$
I^{2 M M}\left(n, \mathbf{t}_{\infty}^{(p)}, \mathbf{t}_{\infty}^{(p)}\right)=\int e^{\operatorname{tr} M_{1}^{p}+\operatorname{tr} M_{2}^{p}} e^{-\operatorname{tr} M_{1} M_{2}} d M_{1} d M_{2}
$$

факторизуется в произведение $p$ бесконечносолитонных тау-функций.

4.3. Одноматричная модель. Статсумма одноматричной модели есть

$$
Z\left(N, g, g_{4}\right)=\int d M \exp \left(-N \frac{g}{2} \operatorname{tr} M^{2}-N \sum_{m>2} \frac{g_{2 m}}{2 m} \operatorname{tr} M^{2 m}\right)
$$

где $M$ - эрмитова $(N \times N)$-матрица, а мера определена как

$$
d M=\prod_{i=1}^{n} d M_{i i} \prod_{i<j}^{n} d \operatorname{Re} M_{i j} d \operatorname{Im} M_{i j} .
$$

Для простоты в модели (4.16) мы взяли четный потенциал. 
Как следует из работы [6], а также из приложения Б, ряд теории возмущений факторизуется:

$$
\begin{aligned}
Z\left(2 N, g, g_{4}\right) & =\tau_{r^{(1)}}\left(N, \mathbf{t}, \mathbf{t}^{*}\right) \tau_{r^{(2)}}\left(N, \mathbf{t}, \mathbf{t}^{*}\right), \\
Z\left(2 N+1, g, g_{4}\right) & =\tau_{r^{(1)}}\left(N+1, \mathbf{t}, \mathbf{t}^{*}\right) \tau_{r^{(2)}}\left(N, \mathbf{t}, \mathbf{t}^{*}\right),
\end{aligned}
$$

где

$$
\mathbf{t}=\left(0,-\frac{N g_{4}}{4},-\frac{N g_{6}}{6},-\frac{N g_{8}}{8}, \ldots\right), \quad \mathbf{t}^{*}=\left(\frac{1}{2 N g}, 0,0,0, \ldots\right)=\frac{1}{2 N g} \mathbf{t}_{\infty} .
$$

Каждый множитель в (4.17) удовлетворяет уравнению (2.35) и имеет вид бесконечносолитонного решения. С помощью (4.17), (4.18) найдем

$$
r^{(1)}(k)=2 k(2 k-1), \quad r^{(2)}(k)=(2 k+1)(2 k),
$$

что с учетом формулы (А.21) позволяет определить $T_{m}$ в (2.1) для тау-функций $\tau_{r^{(1)}}\left(N+1, \mathbf{t}, \mathbf{t}^{*}\right)$ и $\tau_{r^{(2)}}\left(N, \mathbf{t}, \mathbf{t}^{*}\right)$.

ЗАмечАниЕ 4 . В случае $t_{k}^{*}=\delta_{k, 4}$ ряд $\tau_{r(1,2)}\left(N, \mathbf{t}, \mathbf{t}^{*}\right)$ может быть получен как ряд теории возмущений для матричного интеграла

$$
\tau_{r^{(1,2)}}\left(N, \mathbf{t}, \mathbf{t}^{*}\right)=C_{N} \int \exp \left(-\frac{\operatorname{tr} M^{2}}{2 N g_{4}}\right) \operatorname{det}\left(1-\frac{\sqrt{-2} M}{g N}\right)^{-N \pm \frac{1}{2}} d M,
$$

в котором интегрирование ведется по эрмитовым $(N \times N)$-матрицам $M, C_{N}$ - нормировочный множитель. Ряд теории возмущений для интеграла (4.21) также есть ряд для бесконечносолитонной тау-функции.

ПРИЛОЖЕНИЕ А

\section{Фермионное представление. Гипергеометрическая тау-функция ЦТ}

Мы будем использовать фермионное представление работ [16], [30] (см. также [18]).

ГТФ иерархии КП в форме (1.19) имеет следующее фермионное представление [1] (некоторые детали вывода выписаны в [6]):

$$
\tau_{r}\left(n, \mathbf{t}, \mathbf{t}^{*}\right)=\left\langle n\left|e^{H(\mathbf{t})} e^{-A\left(\mathbf{t}^{*}\right)}\right| n\right\rangle,
$$

где

$$
\begin{gathered}
H(\mathbf{t})=\sum_{m=1}^{\infty} H_{m} t_{m}, \quad A\left(\mathbf{t}^{*}\right)=\sum_{m=1}^{\infty} A_{m} t_{m}^{*}, \\
H_{m}=\sum_{k=-\infty}^{\infty} \psi_{k} \psi_{k+m}^{*}, \quad m \neq 0, \\
A_{m}=-\sum_{n=-\infty}^{\infty} r(n) \ldots r(n-m+1) \psi_{n} \psi_{n-m}^{*}, \quad m=1,2, \ldots
\end{gathered}
$$


Здесь $\left\{\psi_{m}\right\},\left\{\psi_{m}^{*}\right\} \quad(m \in \mathbb{Z})$ - это фермионные операторы, удовлетворяющие каноническим антикоммутационным соотношениям

$$
\left[\psi_{j}, \psi_{k}^{*}\right]_{+}=\delta_{j k}, \quad\left[\psi_{j}, \psi_{k}\right]_{+}=0, \quad\left[\psi_{j}^{*}, \psi_{k}^{*}\right]_{+}=0, \quad j, k \in \mathbb{Z} .
$$

Вакуумные состояния заряда $n$ в формуле (А.1) определены как

$$
|n\rangle:=\psi_{n-1} \ldots \psi_{1} \psi_{0}|0\rangle, \quad n>0, \quad|n\rangle:=\psi_{n}^{*} \ldots \psi_{-1}^{*}|0\rangle, \quad n<0,
$$

где вакуумный вектор $|0\rangle$ характеризуется свойством

$$
\psi_{m}|0\rangle=0, \quad m<0 ; \quad \psi_{m}^{*}|0\rangle=0, \quad m \geqslant 0 .
$$

Из (А.3) и (А.4) можно получить, что при любых $n, m=1,2, \ldots$ имеют место соотношения $\left[H_{n}, H_{m}\right]=\left[A_{n}, A_{m}\right]=0,\left[H_{n}, A_{m}\right] \neq 0$.

Определим также

$$
H^{*}\left(\mathbf{t}^{*}\right)=\sum_{m=1}^{\infty} H_{-m} t_{m}^{*}
$$

где $H_{-m}, \quad m=1,2, \ldots$, заданы формулой (А.3). Тогда, как легко заметить, для $r \equiv 1$ имеем $A\left(\mathbf{t}^{*}\right)=H^{*}\left(\mathbf{t}^{*}\right)$.

Непосредственно из определений можно вывести, что для любых $\mathbf{t}^{*}$ и $\mathbf{t}$

$$
\left\langle n\left|H^{*}\left(\mathbf{t}^{*}\right)=0, \quad H(\mathbf{t})\right| n\right\rangle=0 .
$$

Приведем также теорему Вика, которая используется при выводе формул для резонансных солитонных решений (именно, при выводе формулы (2.23) из предыдущей формулы (2.22)) и при выводе аналогичной формулы для многокомпонентного случая.

Нам потребуется следующая форма теоремы Вика:

$$
\left\langle n\left|w_{1} \ldots w_{k} w_{1}^{*} \ldots w_{k}^{*}\right| n\right\rangle=\operatorname{det} A, \quad A_{i j}=\left\langle n\left|w_{i} w_{j}^{*}\right| n\right\rangle, \quad i, j=1, \ldots, k,
$$

где $w_{i}, i=1,2, \ldots, k,-$ любые линейные комбинации фермионных операторов $\psi_{m}$, $m \in \mathbb{Z}$, а $w_{i}^{*}, \quad i=1,2, \ldots, k$ - любые линейные комбинации фермионных операторов $\psi_{m}^{*}, m \in \mathbb{Z}$.

Для построения солитонных решений введем

$$
\psi(z)=\sum_{m=-\infty}^{+\infty} \psi_{m} z^{m}, \quad \psi(z)^{*}=\sum_{m=-\infty}^{+\infty} \psi_{m}^{*} z^{-m-1} .
$$

Используя формулу (А.10), получим

$$
\begin{aligned}
\langle n| \psi\left(z_{1}\right) & \ldots \psi\left(z_{k}\right) \psi^{*}\left(z_{-1}\right) \ldots \psi^{*}\left(z_{-k}\right)|n\rangle= \\
& =\left(\frac{z_{1}}{z_{-1}}\right)^{n} \ldots\left(\frac{z_{k}}{z_{-k}}\right)^{n} \frac{\prod_{i<j}^{k}\left(z_{i}-z_{j}\right)\left(z_{-i}-z_{-j}\right)}{\prod_{i, j=1}^{k}\left(z_{i}-z_{-j}\right)} .
\end{aligned}
$$


Для того чтобы получить множители $e^{\xi}$ в $(2.22)$, воспользуемся формулами из работ [16], [18]:

$$
\begin{gathered}
e^{H(\mathbf{t})} \psi(z) e^{-H(\mathbf{t})}=\psi(z) e^{\xi(\mathbf{t}, z)}, \\
e^{H(\mathbf{t})} \psi^{*}(z) e^{-H(\mathbf{t})}=\psi^{*}(z) e^{-\xi(\mathbf{t}, z)}, \\
e^{-H^{*}\left(\mathbf{t}^{*}\right)} \psi(z) e^{H^{*}\left(\mathbf{t}^{*}\right)}=\psi(z) e^{-\xi\left(\mathbf{t}^{*}, z^{-1}\right)}, \\
e^{-H^{*}\left(\mathbf{t}^{*}\right)} \psi^{*}(z) e^{H^{*}\left(\mathbf{t}^{*}\right)}=\psi^{*}(z) e^{\xi\left(\mathbf{t}^{*}, z^{-1}\right)},
\end{gathered}
$$

где

$$
\xi(\mathbf{t}, z)=\sum_{m=1}^{\infty} z^{m} t_{m}
$$

а также соотношениями

$$
\left\langle n\left|e^{H^{*}\left(\mathbf{t}^{*}\right)}=\left\langle n\left|, \quad e^{H(\mathbf{t})}\right| n\right\rangle=\right| n\right\rangle,
$$

которые следуют из (А.9).

Приведем важную формулу [16], [30]. Для $-\beta_{1}<\cdots<-\beta_{k} \leqslant 0 \leqslant \alpha_{k}<\cdots<\alpha_{1}$

$$
\left\langle 0\left|e^{H(\mathbf{t})} \psi_{-\beta_{1}-1}^{*} \ldots \psi_{-\beta_{k}-1}^{*} \psi_{\alpha_{k}} \ldots \psi_{\alpha_{1}}\right| 0\right\rangle=(-1)^{\beta_{1}+\cdots+\beta_{k}+k} s_{(\alpha \mid \beta)}(\mathbf{t}) .
$$

Рассмотрим ЦТ [18], [30]. Наши обозначения $n, \mathbf{t}, \mathbf{t}^{*}$ соответствуют обозначениям $s, x,-y$ работы [18].

В фермионном представлении рассмотрим тау-функцию (1.1), зависящую от трех наборов переменных $\mathbf{t}, T, \mathbf{t}^{*}$ и дискретной переменной $n$ :

$$
\tau\left(n, \mathbf{t}, T, \mathbf{t}^{*}\right)=\left\langle n\left|e^{H(\mathbf{t})} \exp \left(\sum_{-\infty}^{\infty} T_{m}: \psi_{m}^{*} \psi_{m}:\right) e^{H^{*}\left(\mathbf{t}^{*}\right)}\right| n\right\rangle,
$$

где $: \psi_{m}^{*} \psi_{m}:=\psi_{m}^{*} \psi_{m}-\left\langle 0\left|\psi_{m}^{*} \psi_{m}\right| 0\right\rangle$. В уравнениях ЦТ и КП величины $T_{n}$ имеют смысл интегралов движения. Множитель $c_{n}(T)$ служит для нормировки тауфункции: $\tau(n, 0, T, 0)=1$. Это дает

$$
\begin{aligned}
& c_{n}(T)=\tau(n, \mathbf{0}, T, \mathbf{0})=e^{T_{n-1}+\cdots+T_{1}+T_{0}}, \quad n>0, \\
& c_{n}(T)=\tau(n, \mathbf{0}, T, \mathbf{0})=e^{-T_{n}-\cdots-T_{-2}-T_{-1}}, \quad n<0 .
\end{aligned}
$$

Если выполнено равенство

$$
r(m)=e^{T_{m-1}-T_{m}}, \quad m \in \mathbb{Z},
$$

то

$$
c_{n}(T) \tau_{r}\left(n, \mathbf{t}, \mathbf{t}^{*}\right)=\tau\left(n, \mathbf{t}, T, \mathbf{t}^{*}\right) .
$$

Гипергеометрические функции (В.13), (В.21), (В.17), (В.10), перечисленные в приложении В, есть тау-функции (А.18), взятые при определенных значениях времен $n, \mathbf{t}, T, \mathbf{t}^{*}$, см. [1]. 
Следует заметить, что при некоторых специальных значениях переменных $T$ тауфункции (А.18) рассматривались в работах [13] в контексте изучения струн с $c=1$.

Тау-функция иерархии КП $\tau(n)=\tau_{r}\left(n, \mathbf{t}, \mathbf{t}^{*}\right)$, рассматриваемая как функция $n$, подчиняется уравнению Хироты

$$
\tau(n) \partial_{t_{1}^{*}} \partial_{t_{1}} \tau(n)-\partial_{t_{1}} \tau(n) \partial_{t_{1}^{*}} \tau(n)=r(n) \tau(n-1) \tau(n+1),
$$

которое эквивалентно уравнению ЦТ, записанному в следующей форме:

$$
\partial_{t_{1}} \partial_{t_{1}^{*}} \phi_{n}=r(n) e^{\phi_{n-1}-\phi_{n}}-r(n+1) e^{\phi_{n}-\phi_{n+1}},
$$

где

$$
\phi_{n}\left(\mathbf{t}, \mathbf{t}^{*}\right)=-\ln \frac{\tau_{r}\left(n+1, \mathbf{t}, \mathbf{t}^{*}\right)}{\tau_{r}\left(n, \mathbf{t}, \mathbf{t}^{*}\right)} .
$$

Уравнения (А.23) и (А.24) остаются верными и в том случае, когда функция $r$ имеет нули, и соотношения (А.21) выполняется лишь в смысле предельного перехода. В случае, когда $r(n)$ не обращается в нуль, можно сделать замену переменных

$$
\varphi_{n}=-\phi_{n}-T_{n},
$$

которая превращает уравнение (А.24) в стандартное уравнение ЦТ (1.4) [18]. Разница между этим уравнением и уравнением (А.24) возникает из-за нормировочного множителя $c_{n}(T)$.

\section{ПРИЛОЖЕНИЕ Б}

\section{Многокомпонентный случай}

УтвЕРЖДЕНИЕ 1. Пусть $p$ - положительное челое число. Для каждого $i=$ $1,2, \ldots, p$ имеет место равенство

$$
\tau_{r}\left(n p+i, \mathbf{t}^{[p]}, \mathbf{t}^{*[p]}\right)=\tau_{r^{(1)}}\left(n+1, \mathbf{t}, \mathbf{t}^{*}\right) \ldots \tau_{r^{(i)}}\left(n+1, \mathbf{t}, \mathbf{t}^{*}\right) \tau_{r^{(i+1)}}\left(n, \mathbf{t}, \mathbf{t}^{*}\right) \ldots \tau_{r^{(p)}}\left(n, \mathbf{t}, \mathbf{t}^{*}\right)
$$

где каждая тау-функция $\tau_{r(m)}\left(j, \mathbf{t}, \mathbf{t}^{*}\right)($ при $j=n, n+1)$ задана формулой (A.1), в которой $r$ определяется с помощъю соотношений

$$
\begin{gathered}
r^{(m)}(k)=R(k p+m-1), \quad m=1, \ldots, p, \quad k \in \mathbb{Z}, \\
R(k)=r(k) r(k-1) \ldots r(k-p+1) .
\end{gathered}
$$

Времена $\mathbf{t}^{[p]}, \mathbf{t}^{*[p]}$ в левой части равенства (Б.1) связаны с временами $\mathbf{t}, \mathbf{t}^{*}$ в правой части этого равенства с помощъю формул (3.6).

ДокАзАтЕльство. Введем, следуя работе [16], иветные фермионы, которые получаются в результате следующей перенумерации исходных фермионов: $\psi_{k}^{(m)}:=$ $\psi_{k p+m-1}, \quad \psi_{k}^{*(m)}:=\psi_{k p+m-1}^{*}, m=1, \ldots, p$ и $k \in \mathbb{Z}$. Ясно, что для всех $j, k$

$$
\left[\psi_{j}^{(m)}, \psi_{k}^{*(n)}\right]_{+}=\left[\psi_{j}^{(m)}, \psi_{k}^{(n)}\right]_{+}=\left[\psi_{j}^{*(m)}, \psi_{k}^{*(n)}\right]_{+}=0, \quad m \neq n
$$


Тогда вакуумное среднее (А.1) распадается в произведение, состоящее из $p$ вакуумных средних от фермионов цвета $1, \ldots, p$.

Пусть $\left(\alpha_{1}, \alpha_{2}, \ldots \mid \beta_{1}, \beta_{2}, \ldots\right)$ - обозначение Фробениуса для разбиения $\lambda$. Положим

$$
r_{\lambda}^{(m)}(k)=r_{\tilde{\lambda}}(k+m-p+1), \quad m=1, \ldots, p,
$$

где $\tilde{\lambda}$ есть разбиение $\left(p \alpha_{1}, p \alpha_{2}, \ldots \mid p \beta_{1}, p \beta_{2}, \ldots\right)$.

Рассмотрим набор, состоящий из разбиений $\lambda^{(m)}=\left(\alpha_{1}^{(m)}, \alpha_{2}^{(m)}, \ldots, \alpha_{k^{(m)}}^{(m)} \mid \beta_{1}^{(m)}\right.$, $\left.\beta_{2}^{(m)}, \ldots, \beta_{k^{(m)}}^{(m)}\right), \quad m=1, \ldots, p$. Припишем числам $\left\{\alpha_{k_{i}}^{(m)} p+m-1 \mid i=1, \ldots, k^{(m)}\right.$, $m=1, \ldots, p\}$ нижние индексы так, чтобы $\alpha_{1}>\alpha_{2}>\cdots>\alpha_{K}>0$, где $K=k^{(1)}+$ $\cdots+k^{(p)}$. В силу самой конструкции убывание имеет строгий характер. Аналогично, рассмотрим числа $\left\{\beta_{k_{i}}^{(m)} p+m-p \mid i=1, \ldots, k^{(m)}, m=1, \ldots, p\right\}$ и также перенумеруем их в порядке убывания: $\beta_{1}>\beta_{2}>\cdots>\beta_{K}>0$. Рассмотрим разбиение $\lambda=$ $\left(\alpha_{1}, \ldots, \alpha_{K} \mid \beta_{1}, \ldots, \beta_{K}\right)$. Используя фермионное представление (А.17), получим

$$
\prod_{m=1}^{p} s_{\lambda^{(m)}}(\mathbf{t})=(-1)^{c_{\lambda}} s_{\lambda}\left(\mathbf{t}^{[p]}\right), \quad s_{\lambda}(0, \ldots, 0,1,0, \ldots, 0,0, \ldots)=(-1)^{c_{\lambda}} \prod_{m=1}^{p} H_{\lambda^{(m)}}^{-1},
$$

где знаковый множитель $(-1)^{c_{\lambda}}$ возникает из-за переупорядочивания фермионов внутри вакуумного среднего. Именно такой множитель возникает при доказательстве теоремы 2 в результате переупорядочивания фермионов в выражении (3.1), когда мы получаем ряд по числам $a_{i j}^{m n}$.

ПРИЛОЖЕНИЕ В

\section{Задачи, в которых встречается ГТФ}

В данном разделе мы укажем соответствие между ГТФ и рядами, рассматриваемыми в некоторых работах.

1. Двумерная теория Янга-Миллса [31] и частные случаи так называемой нелокальной двумерной теорией Янга-Миллса [32], рассматриваемые на диске, приводят к следующему выражению для коррелятора двух петель Вильсона:

$$
Z\left(U_{1}, U_{2}\right)=\sum_{R} \xi_{R}\left(U_{1}^{-1}\right) \xi_{R}\left(U_{2}^{-1}\right) e^{A \sum_{m=1}^{\infty} a_{m} C_{m}(R)} .
$$

Здесь $a_{m}$ - некоторые константы (в 2D-теории Янга-Миллса все $a_{m}$ равны нулю, кроме константы $a_{2}$, которая просто выражается через константу связи теории [31]). Суммирование ведется по всем неприводимым представлениям калибровочной группы. Матрицы $U_{1}$ и $U_{2}$ - это упорядоченные экспоненты (петли Вильсона) от интегралов по калибровочному полю по границам диска. Число $A$ - это площадь диска, a $C_{m}, m=1,2, \ldots,-$ значения операторов Казимира для калибровочной группы $S U(N)$ :

$$
C_{m}(R)=\sum_{i=1}^{N}\left[\left(\lambda_{i}+N-i\right)^{m}-(N-i)^{m}\right]
$$


Положив

$$
m t_{m}=\operatorname{tr} U_{1}^{m}, \quad m t_{m}^{*}=\operatorname{tr} U_{2}^{m},
$$

мы получим, что ряд (В.1) совпадает с (1.1).

2. Производящая функщия для двойных чисел Гурвища введена в работе [10] при исследовании потенциала Громова-Виттена. Она также имеет форму (1.1),

$$
\tau\left(P, P^{\prime}, \beta, q\right)=\sum_{\lambda} q^{|\lambda|} e^{\beta f_{2}(\lambda)} s_{\lambda}(P) s_{\lambda}\left(P^{\prime}\right),
$$

где $P$ и $P^{\prime}$ можно отождествить соответственно с $\mathbf{t}$ и $\mathbf{t}^{*}$, использованными в данной работе, и где

$$
f_{2}(\lambda)=\frac{1}{2} \sum_{i}\left[\left(\lambda_{i}-i+\frac{1}{2}\right)^{2}-\left(-i+\frac{1}{2}\right)^{2}\right] .
$$

Выбирая $\mathbf{t}=\mathbf{t}^{*}=e^{u / 2} \mathbf{t}_{\infty}:=\left(e^{u / 2}, 0,0, \ldots\right)$ (как это проделано в [10], [11]), можно получить следующую запись одномерной ЦТ (1.22):

$$
e^{-u} \frac{d^{2} \phi_{n}}{d u^{2}}=r(n) e^{\phi_{n-1}-\phi_{n}}-r(n+1) e^{\phi_{n}-\phi_{n+1}} .
$$

После редукции $n=\ln t_{1} t_{1}^{*}+$ const, сходной с редукцией КП к уравнению Бенеджамино-Оно [33], получим так называемое эквивариантное уравнение ЦТ [10]. В этом случае в уравнении $(2.32) \tilde{t}_{2}=\beta$, а все остальные $\tilde{t}_{k}=0$.

3. Суперсимметричные калибровочные теории [8], [9]. В работе [8] рассматривалась слабо нарушенная суперсимметричная $(\mathcal{N}=4)$-теория, которая сводится к суперсимметричной калибровочной $(\mathcal{N}=2)$-теории с калибровочной группой $U(N)$. Подсчет функционального интеграла в случае $U(1)$ в четырехмерном евклидовом пространстве на так называемом $\Omega$-фоне приводит к выражению

$$
Z(\Lambda)=e^{-1 / 12} \sum_{\mathbf{k}} \Lambda^{2|\mathbf{k}|} \mu(\mathbf{k})^{2}, \quad \mu(\mathbf{k})=\prod_{i<j}\left(\frac{k_{i}-k_{j}+j-i}{j-i}\right) .
$$

Переобозначим $\mathbf{k}=\left(k_{1}, k_{2}, \ldots\right)$ как $\lambda=\left(\lambda_{1}, \lambda_{2}, \ldots\right), \Lambda^{2}=r$. Можно видеть, что тогда ряд в (В.7) является тау-функцией гипергеометрического типа, где $\mathbf{t}=\mathbf{t}^{*}=\mathbf{t}_{\infty}$. Ряд для случая $N>1$ после некоторого преобразования также приводится к виду (1.1). В [8] рассматривалась компактифицированная на окружность суперсимметричная 5D-теория с восемью суперзарядами. Для случая $U(1)$ подсчет функционального интеграла дает

$$
\begin{gathered}
Z(\beta, \Lambda)=\Lambda^{\left(1-N^{2}\right) / 12} e^{\gamma_{\hbar}(0 \mid \beta, \Lambda)} \sum_{\mathbf{k}}(\beta \Lambda)^{2|\mathbf{k}|} \mu(\mathbf{k})^{2}, \\
\mu(\mathbf{k})=\prod_{i<j}\left(\frac{\operatorname{sh}\left(\beta \hbar\left(k_{i}-k_{j}+j-i\right) / 2\right)}{\operatorname{sh}(\beta \hbar(j-i) / 2)}\right)
\end{gathered}
$$

где $\beta$ - параметр теории, связанный с компактификацией. Это есть тау-функция (1.6), в которой $\mathbf{t}=\mathbf{t}^{*}=\mathbf{t}(\infty, q)$. 
4. Производящая функция для индексов пересечения на схемах Гильберта была выписана в [12]. Этот ряд также является ГТФ. Обозначения $m, t, s$ и $x$ из работы [12] соответствуют нашим обозначениям $n, \mathbf{t}, \mathbf{t}^{*}$ и $T$, соответственно. Функция $\tau(t, s, x, m)$ работы [12] соответствует нашей функции $\tau\left(n, \mathbf{t}, T, \mathbf{t}^{*}\right)$ и является производящей функцией эквивариантных индексов пересечения на схемах Гильберта точек аффинной плоскости.

5. Один из примеров точно решаемых матричных моделей приведен в работе [7]. Пусть

$$
\begin{array}{r}
\int_{U(n)} \operatorname{det} U^{\mp m} \operatorname{det}(1-X U)^{-a} \operatorname{det}\left(1-U^{-1} Y\right)^{-b} d_{*} U= \\
=\frac{1}{(n)_{\sigma}}\left\{\begin{array}{c}
(a)_{\sigma} \operatorname{det} X^{m} \\
(\tilde{a})_{\sigma} \operatorname{det} Y^{m}
\end{array}\right\}{ }_{2} F_{1}\left(\begin{array}{c}
\tilde{a}, \tilde{b} \\
n+m
\end{array} \mid X Y\right),
\end{array}
$$

где $\sigma$ обозначает разбиение $(m, \ldots, m), \quad \ell(\sigma)=n, \quad m \geqslant 0, \quad \tilde{a}=a+m, \quad \tilde{b}=b$ для верхнего знака, $\tilde{a}=a, \tilde{b}=b+m$ для нижнего знака в левой части (В.9). Обозначение $(n)_{\sigma},(a)_{\sigma}$ см. ниже в (В.12). Гауссова гипергеометрическая функция матричного аргумента ${ }_{2} F_{1}$ приводится в (В.10), все гипергеометрические функции матричного аргумента являются примерами ГТФ. При $m=0,-a=-b=p \in$ $\mathbb{Z}_{\geqslant 0}$ интеграл (В.9) был недавно подсчитан методом реплик в форме детерминанта матрицы размера $(p \times p)[34]$.

Отметим, что ${ }_{2} F_{1}(a, b ; c \mid X)$ с целыми $a, b, c$ является решением уравнения Пенлеве $\mathrm{V}[35]$.

6. Гипергеометрические функиии матричного аргумента $X$ (X- нормальная матрица с собственными значениями $\left.\mathbf{x}^{N}=x_{1}, \ldots, x_{N}\right)$ определяются формулой [3]

$$
{ }_{p} F_{s}\left(\begin{array}{c}
a_{1}, \ldots, a_{p} \\
b_{1}, \ldots, b_{s}
\end{array} \mid X\right)=\sum_{\substack{\lambda \\
l(\lambda) \leqslant N}} \frac{\left(a_{1}\right)_{\lambda} \ldots\left(a_{p}\right)_{\lambda}}{\left(b_{1}\right)_{\lambda} \ldots\left(b_{s}\right)_{\lambda}} \frac{s_{\lambda}\left(\mathbf{x}^{N}\right)}{H_{\lambda}}
$$

где суммирование ведется по всем разбиениям. В этой формуле использованы следующие обозначения: $H_{\lambda}$ - произведение длин крюков,

$$
\begin{gathered}
H_{\lambda}=\prod_{(i, j) \in \lambda} h_{i j}, \quad h_{i j}=\left(\lambda_{i}+\lambda_{j}^{\prime}-i-j+1\right), \\
(a)_{\lambda}=(a)_{\lambda_{1}}(a-1)_{\lambda_{2}} \ldots(a-k+1)_{\lambda_{k}}, \quad(a)_{\mathbf{0}}=1 .
\end{gathered}
$$

При $N=1$ мы получаем

$$
{ }_{p} F_{s}\left(a_{1}, \ldots, a_{p} ; b_{1}, \ldots, b_{s} ; x\right)=\sum_{n=0}^{\infty} \frac{\left(a_{1}\right)_{n} \ldots\left(a_{p}\right)_{n}}{\left(b_{1}\right)_{n} \ldots\left(b_{s}\right)_{n}} \frac{x^{n}}{n !}
$$


В работах [1] показано, что (В.10) является тау-функцией (1.19):

$$
{ }_{p} F_{s}\left(\begin{array}{c}
a_{1}+n, \ldots, a_{p}+n \\
b_{1}+n, \ldots, b_{s}+n
\end{array} \mid X\right)=\tau_{r}\left(n, \mathbf{t}, \mathbf{t}_{\infty}\right),
$$

где

$$
\begin{gathered}
r(k)=\prod_{i=1}^{p}\left(k+a_{i}\right) \prod_{i=1}^{s}\left(k+b_{i}\right)^{-1}, \\
t_{m}=\operatorname{tr} \mathbf{X}^{m}, \quad m=1,2,3, \ldots .
\end{gathered}
$$

Так как в качестве аргумента тау-функции в правой части (В.14) стоит $\mathbf{t}_{\infty}$, то в силу (2.32) гипергеометрические функции матричного аргумента всегда имеют солитонную трактовку.

Пусть $|q|<1$. Ряд

$$
{ }_{p} \Phi_{s}\left(a_{1}, \ldots, a_{p} ; b_{1}, \ldots, b_{s} ; q, \mathbf{x}^{N}\right)=\sum_{\substack{\lambda \\ l(\lambda) \leqslant N}} \frac{\left(q^{a_{1}} ; q\right)_{\lambda} \ldots\left(q^{a_{p}} ; q\right)_{\lambda}}{\left(q^{b_{1}} ; q\right)_{\lambda} \ldots\left(q^{b_{s}} ; q\right)_{\lambda}} \frac{q^{n(\lambda)}}{H_{\lambda}(q)} s_{\lambda}\left(\mathbf{x}^{N}\right),
$$

где суммирование ведется по всем разбиениям, называется $q$-деформированной гипергеометрической функцией матричного аргумента [36]. Далее,

$$
H_{\lambda}(q)=\prod_{(i, j) \in \lambda}\left(1-q^{h_{i j}}\right), \quad h_{i j}=\left(\lambda_{i}+\lambda_{j}^{\mathrm{tr}}-i-j+1\right),
$$

где $\lambda^{\operatorname{tr}}$ обозначает сопряженное разбиение [22],

$$
\begin{gathered}
\left(q^{c} ; q\right)_{\lambda}=\left(q^{c} ; q\right)_{\lambda_{1}}\left(q^{c-1} ; q\right)_{\lambda_{2}} \ldots\left(q^{c-k+1} ; q\right)_{\lambda_{k}}, \\
q^{n(\lambda)}=q^{\sum_{i=1}^{k}(i-1) \lambda_{i}} .
\end{gathered}
$$

Для $N=1$ получим обычную $q$-деформированную гипергеометрическую функцию

$$
{ }_{p} \Phi_{s}\left(a_{1}, \ldots, a_{p} ; b_{1}, \ldots, b_{s} ; q, x\right)=\sum_{n=0}^{\infty} \frac{\left(q^{a_{1}} ; q\right)_{n} \ldots\left(q^{a_{p}} ; q\right)_{n}}{\left(q^{b_{1}} ; q\right)_{n} \ldots\left(q^{b_{s}} ; q\right)_{n}} \frac{x^{n}}{(q ; q)_{n}}
$$

В работах [1] найдено, что (В.17) является тау-функцией (1.19):

$$
{ }_{p} \Phi_{s}\left(a_{1}, \ldots, a_{p} ; b_{1}, \ldots, b_{s} ; q, X\right)=\tau_{r}(n, \mathbf{t}, \mathbf{t}(\infty, q)),
$$

где

$$
\begin{gathered}
t_{m}=\operatorname{tr} X^{m}, \quad m=1,2,3, \ldots, \\
r(n)=\frac{\prod_{i=1}^{p}\left(1-q^{a_{i}+n}\right)}{\prod_{i=1}^{s}\left(1-q^{b_{i}+n}\right)},
\end{gathered}
$$

$\mathbf{t}(\infty, q)$ задается в формуле (2.5). Таким образом, в силу (2.38) гипергеометрическая функция (В.17) является солитонной тау-функцией. 
Благодарности. Автор благодарен А. Забродину, А. Лосеву, С. Натанзону, И. Луценко и особенно Н. Некрасову за полезные обсуждения. Автор также благодарен М. Бойти, Т. Дегасперису, П. Сантини, Г. Хельминку и Й. ван де Леру за обсуждения и организацию лекции в Галиполли, семинаров в университетах Рима, Утрехта и Твенте, где начата данная работа. Работа поддержана РФФИ (грант № 02-02-17382) и программой РАН "Математические методы нелинейной динамики".

\section{Список литературы}

[1] A. Yu. Orlov, D. M. Scherbin. Fermionic representation for basic hypergeometric functions related to Schur polynomials. nlin.SI/0001001; Physica. D. 2001. V. 152-153. P. 51.

[2] A. Yu. Orlov, D. M. Scherbin. J. Phys. A. 2001. V. 34. P. 2295.

[3] K. I. Gross, D. S. Richards. Trans. Amer. Math. Soc. 1987. V. 301. P. 781; H. Я. Buленкин, A. У. Климык. Представления групп Ли и специальные функции. В сб.: Итоги науки и техники. Сер. Современные проблемы математики. Фундаментальные направления. Т. 59. Ред. А. А. Кириллов. М.: ВИНИТИ, 1990.

[4] A. Yu. Orlov. Acta Appl. Math. 2005. V. 86. № 1-2. P. 131; nlin.SI/0207030.

[5] A. Yu. Orlov. Tau functions and matrix integrals. math-ph/0210012.

[6] Дж. Харнад, А. Ю. Орлов. ТМФ. 2003. Т. 137. № 3. С. 375; nlin.SI/0211051.

[7] A. Yu. Orlov. Int. J. Mod. Phys. A. 2004. V. 19. P. 276; nlin.SI/0209063.

[8] N. A. Nekrasov. Adv. Theor. Math. Phys. 2004. V. 7. P. 831; hep-th/0206161.

[9] A. S. Losev, A. Marshakov, N. A. Nekrasov. Small instantons, little strings and free fermions. Preprint ITEP-TH-1-03. Moscow: ITEP, 2003; hep-th/0302191.

[10] A. Okounkov. Math. Res. lett. 2000. V. 7. P. 447; math.AG/0004128.

[11] A. Okounkov, R. Pandharipande. The equivariant Gromov-Witten theory of $P^{1}$. math.AG/0207233.

[12] W.-P. Li, Z. Qin, W. Wang. Int. Math. Res. Notices. 2004. V. 40. P. 2085; math.AG/0302211.

[13] T. Nakatsu, K. Takasaki, S. Tsujimaru. Nucl. Phys. B. 1995. V. 443. P. 155; hepth/9501038; T. Takasaki. Commun. Math. Phys. 1996. V. 181. P. 131; hep-th/9506089.

[14] A. Yu. Orlov. Hypergeometric tau functions $\tau\left(\mathbf{t}, T, \mathbf{t}^{*}\right)$ as $\infty$-soliton tau function in $T$ variables. nonlin.SI/0305001.

[15] В. Е. Захаров, А. Б. Шабат. Функц. анализ и его прилож. 1974. Т. 8. № 3. С. 45.

[16] M. Jimbo, T. Miwa. Publ. RIMS Kyoto Univ. 1983. V. 19. P. 943.

[17] А. В. Михайлов. Письма в ЖЭТФ. 1979. Т. 30. С. 443.

[18] K. Ueno, K. Takasaki. Adv. Stud. Pure Math. 1984. V. 4. P. 1.

[19] В. Е. Захаров, С. В. Манаков, С. П. Новиков, Л. П. Питаевский. Теория солитонов. Метод обратной задачи. М.: Наука, 1980.

[20] K. Takasaki. Adv. Stud. Pure Math. 1984. V. 4. P. 139.

[21] А. Ю. Орлов, Д. М. Щербин. ТМФ. 2001. Т. 128. № 1. С. 84.

[22] I. G. Macdonald. Symmetric Functions and Hall Polynomials. Oxford: Clarendon Press, 1995.

[23] A. Mironov. Seiberg-Witten theory and duality in integrable systems. Preprint FIAN-TD29-00. Moscow: FIAN, 2003; ITEP-TH-63-00. Moscow: ITEP, 2000; hep-th/0011093. 
[24] Yu. Yu. Berest, I. M. Loutsenko. Commun. Math. Phys. 1997. V. 190. P. 113-132; solv-int/9704012; I. M. Loutsenko, V. Spiridonov. J. Statist. Phys. 2000. V. 99. P. 751 ; cond-mat $/ 9909308$.

[25] L.-L. Chau, O. Zaboronsky. Commun. Math. Phys. 1998. V. 196. P. 203; hep-th/9711091.

[26] L.-L. Chau, Y. Yu. Phys. Lett. A. 1992. V. 167. P. 452.

[27] M. Mineev-Weinstein, P. Wiegmann, A. Zabrodin. Phys. Rev. Lett. 2000. V. 84. P. 5106.

[28] А. В. Забродин. ТМФ. 2001. Т. 129. № 2. С. 239.

[29] C. Itzykson, J. B. Zuber. J. Math. Phys. 1980. V. 21. P. 411.

[30] E. Date, M. Jimbo, M. Kashiwara, T. Miwa. Transformation groups for soliton equations. In: Non-linear Integrable Systems - Classical Theory and Quantum Theory. Proc. RIMS. Symp. (Kyoto, Japan, May 13-16, 1981). Eds. M. Jimbo, T. Miwa. Singapore: World Scientific, 1983. P. 39.

[31] А. Б. Мигдал. ЖЭТФ. 1975. Т. 69. С. 810.

[32] K. Saaidi, M. Khorrami. Eur. Phys. J. C. 2002. V. 23. P. 757; hep-th/0304204.

[33] D. Lebedev, A. Orlov, S. Pakulyak, A. Zabrodin. Phys. Lett. A. 1991. V. 160. P. 166.

[34] Y. V. Fyodorov, H.-J. Sommers. J. Phys. A. 2003. V. 36. P. 3303; nlin.CD/0207051.

[35] M. Adler, P. van Moerbeke. Adv. Math. 2004. V. 181. P. 190; math.CO/0110281.

[36] N. Ya. Vilenkin, A. U. Klimyk. Representation of Lie Groups and Special Functions, Recent Advances. Kluwer Academic Publishers, 1995; S. C. Milne. Summation theorems for basic hypergeometric series of Schur function argument. In: Progress in Approximation Theory. Proc. Int. Conf. on Approximation Theory (Tampa, South Florida, USA, March 19-22, 1990). Comput. Math. V. 19. Eds. A. A. Gonchar, E. B. Saff. N. Y.: Springer, 1992. P. 51.

Поступила в редакцию 10.I.2004 г., после доработки 10.VI.2005 г. 